\title{
Christian Zimmer*
}

\section{Kasus im Namdeutschen Case in Namdeutsch}

https://doi.org/10.1515/zgl-2020-2004

Abstract: This paper focusses on case marking in informal Namibian German (so called Namdeutsch). Whilst the use of nominative and accusative case is stable and similar to Standard German, there is a considerable amount of variation with regard to the dative case. This phenomenon is analysed in detail using corpus and questionnaire data. Multifactorial analyses reveal that several sociolinguistic and grammatical factors have a significant impact on the language use in this particular domain. Subsequently, the results are compared with other extraterritorial varieties of German and various similarities are found. This supports the idea that there are variety/language overarching principles at work.

$\begin{array}{ll}1 & \text { Einleitung } \\ 2 & \text { Die deutsche Sprache in Namibia } \\ 3 & \text { Korpusdaten } \\ 3.1 & \text { Datenerhebung } \\ 3.2 & \text { Überblick } \\ 3.3 & \text { Analyse } \\ 4 & \text { Fragebogendaten } \\ 4.1 & \text { Datenerhebung } \\ 4.2 & \text { Analyse } \\ 5 & \text { Interpretation der Ergebnisse } \\ 5.1 & \text { Vergleich der Datentypen } \\ 5.2 & \text { Soziolinguistische Variablen } \\ 5.3 & \text { Grammatische Variablen } \\ 6 & \text { Fazit } \\ & \text { Danksagung } \\ & \text { Literatur }\end{array}$

\footnotetext{
*Kontaktperson: Dr. Christian Zimmer: Institut für Deutsche und Niederländische Philologie, Freie Universität Berlin, Habelschwerdter Allee 45, D-14195 Berlin, E-Mail: christian.zimmer@fu-berlin.de
} 


\section{Einleitung}

Variation, Wandel und Verlust im Bereich der Kasusmarkierung extraterritorialer Varietäten des Deutschen gehören zu den variationslinguistischen Themen, die in den vergangenen Jahren besonders ausführlich diskutiert wurden (Boas 2009a: 182). Mit dem vorliegenden Aufsatz möchte ich einen Beitrag zu dieser Diskussion leisten, indem ich mich zwei Forschungslücken widme.

Zum einen wurde das Namdeutsche, der informelle Sprachgebrauch deutschsprachiger NamibierInnen, mit Blick auf die Kasusverwendung bislang noch nicht systematisch beschrieben und analysiert. Die Beschreibung des Namdeutschen scheint aber lohnenswert, da es sich hier um eine extraterritoriale Varietät handelt, die in mehrerlei Hinsicht besonders ist (Wiese et al. 2017). Wie sich weiter unten zeigen wird, hebt sich das Namdeutsche in einigen Bereichen auch nominalmorphologisch von anderen extraterritorialen Varietäten des Deutschen ab. Ein wichtiger Aspekt ist dabei die vergleichsweise große Ähnlichkeit zum Standarddeutschen. Hier stellt sich natürlich die Frage nach dem Warum, deren Beantwortung neues Licht auf die Bedeutung linguistisch relevanter Unterschiede zwischen dem Deutschen in Namibia und anderen extraterritorialen Varietäten des Deutschen zu werfen vermag. Hierzu zählen z. B. der Einfluss der Beschulung oder der Kontakt zu Deutschsprachigen in/aus Europa usw. Darüber hinaus ermöglichen die neu geschaffenen Ressourcen zum Namdeutschen (s. unten) auch die Untersuchung von Faktoren und Prinzipien, die für die allgemeine Diskussion zu Kasusschwankungen und über das Namdeutsche hinaus relevant sind.

Zum anderen handelt es sich bei dem vorliegenden Aufsatz meines Wissens um den ersten Versuch, Kasus-Schwankungen in einer deutschen Kontaktvarietät mit einem inferenzstatistischen, multifaktoriellen Modell zu analysieren. Dieses Vorgehen erscheint angebracht, da in der Literatur häufig betont wird, dass mehrere sich zum Teil überlappende Faktoren relevant sind für die Analyse und Erklärung beobachteter Schwankungen (s. unten). Deren Zusammenspiel kann am besten multifaktoriell modelliert werden. Somit stellt der vorliegende Beitrag den Versuch dar, das Phänomen möglichst umfassend und unter Berücksichtigung einer Vielzahl potentieller Einflussgrößen zu beschreiben und zu analysieren.

Der Aufsatz ist wie folgt gegliedert: Zunächst werden wichtige soziolinguistische Aspekte zum Deutschen in Namibia umrissen (Kapitel 2). Die sich anschließenden Kapitel widmen sich der Erhebung und Analyse zweier unterschiedlicher Datentypen, nämlich Korpusdaten (Kapitel 3) und Fragebogendaten (Kapitel 4). Die mithilfe der unterschiedlichen Daten gewonnenen Analyse-Ergebnisse werden in Kapitel 5 interpretiert, wobei auch Erkenntnisse zu anderen extrater- 
ritorialen Varietäten des Deutschen einbezogen werden. Abschließend wird ein kurzes Fazit gezogen (Kapitel 6).

\section{Die deutsche Sprache in Namibia}

Die deutschsprachige Minderheit in Namibia geht auf die Kolonialisierung des Gebietes (Deutsch-Südwestafrika, 1884-1915) und sich anschließende Migration zurück, die sich bis heute fortsetzt. Derzeit umfasst die deutschsprachige Community etwa 20.000 SprecherInnen. Alleinige Amtssprache ist seit der Unabhängigkeit Namibias im Jahr 1990 das Englische, das allerdings nur von einem sehr geringen Teil der Bevölkerung Erstsprache ist (vgl. z. B. Zimmer 2019). Neben dem Englischen dient auch Afrikaans in einigen Bereichen als Lingua Franca. Die am weitesten verbreitete Erstsprache ist die Bantu-Sprache Oshiwambo. Ebenfalls weit verbreitet sind weitere Bantu- (z. B. Otjiherero) sowie Khoisansprachen (z. B. Khoekhoegowab). Charakteristisch für Namibia ist insgesamt ein hohes Maß an Viel- und Mehrsprachigkeit. Auch die Mitglieder der deutschsprachigen Community sind in aller Regel mindestens dreisprachig und sprechen neben den germanischen Sprachen Deutsch, Englisch und Afrikaans zum Teil auch Bantu- und/ oder Khoisansprachen.

Im öffentlichen Raum ist das Deutsche vergleichsweise präsent, obwohl die deutschsprachige Community nur etwa $1 \%$ der Bevölkerung ausmacht. Das spiegelt zum einen wider, dass das Deutsche eine wichtige Rolle im Tourismus und in der Wirtschaft spielt, ist zum anderen historisch bedingt und hat seine Wurzeln in der damaligen deutschen Kolonialherrschaft (während dieser Zeit war Deutsch auch alleinige Amtssprache). Nachdem das Deutsche Reich im Anschluss an den Ersten Weltkrieg alle Kolonien abtreten musste, ging die Verwaltung des heutigen Namibia an Südafrika über, das Südwestafrika zuvor besetzt hatte (Böhm 2003: 526). In der Folge dessen wurde auch in Südwestafrika bis zur Unabhängigkeit 1990 die Apartheidspolitik durchgesetzt. Während der Apartheidszeit hatte das Deutsche einige Privilegien, war aber keine Amtssprache mehr (zur Rolle der Apartheid beim Spracherhalt des Deutschen in Namibia s. Schweizer 1982). Heute genießt das Deutsche als eine von 13 „Nationalsprachen“ noch immer gewisse Rechte, ist dem Englischen aber klar untergeordnet (vgl. hierzu z. B. Shah \& Zappen-Thomson 2018).

Die deutschsprachige Community gehört nach wie vor $\mathrm{zu}$ den sozioökonomisch am besten gestellten Gruppen im Land. Dieser Umstand erleichtert die Vernetzung und die Sprachpflege, die generell einen hohen Stellenwert in der Community hat (vgl. hierzu auch Zappen-Thomson 2019). So wurden bzw. 
werden z. B. deutschsprachige Medien aus der deutschsprachigen Gemeinschaft heraus finanziell unterstützt (es gibt eine deutschsprachige Tageszeitung, die Allgemeine Zeitung, deutschsprachige Radiosender usw.), Privatschulen werden unterhalten, die das Fach Deutsch als Muttersprache (DaM) anbieten und über das Jahr verteilt finden in vielen Regionen des Landes Events wie Oktoberfeste und Karnevalsveranstaltungen statt.

Dass gleichermaßen Oktoberfeste wie Karnevalsveranstaltungen (nach rheinischer Prägung) im Land organisiert werden, ist dann auch Ausdruck der Diversität der deutschsprachigen ImmigrantInnen. Anders als bei einigen anderen extraterritorialen Varietäten des Deutschen lässt sich für das Namdeutsche nämlich kein klar abgrenzbarer Raum identifizieren, dem alle oder zumindest die deutliche Mehrheit der deutschsprachigen ImmigrantInnen entstammen. Dies illustriert Abbildung 1, die auf den Informationen basiert, die wir im Rahmen der Metadatenerfassung von unseren ProbandInnen sammeln konnten (alle weiteren Infos zur Datenerhebung finden sich weiter unten in Abschnitt 2.1). Die dazugehörigen Fragen sind in (1) wiedergegeben. In aller Regel gaben die ProbandInnen daraufhin nicht nur eine Region, sondern einen ganz konkreten Ort an. Die genannten Dörfer und Städte sind in Abbildung 1 verortet. ${ }^{1}$

(1) Hat Deine Mutter [und daran anschließend: hat Dein Vater] Vorfahren aus Deutschland? Falls ja: Aus welcher Region?

Die Abbildung veranschaulicht, dass im heutigen Namibia EmigrantInnen aus vielen Teilen des deutschsprachigen Gebiets aufeinandertrafen. Man kann demnach davon ausgehen, dass die Deutschsprachigen im heutigen Namibia (zunächst) eine sprachlich relativ heterogene Gruppe darstellten und zahlreiche Varietäten in Kontakt zueinander traten (vgl. Zimmer i. Dr.).

Mit Blick auf die grammatische Variation, die in den folgenden Abschnitten untersucht wird, bedeutet dies, dass es nicht möglich ist, eine diachrone Perspektive einzunehmen, indem der heutige Sprachgebrauch mit historischen Merkmalen einer spezifischen deutschen Varietät verglichen wird. Vielmehr wäre es angebracht, Merkmale vieler verschiedener Varietäten zusammenzustellen und aus der Perspektive des Varietätenkontakts mit dem heutigen Sprachstand

1 Die Karte wurde mit dem REDE SprachGIS erstellt (Schmidt, Herrgen \& Kehrein 2008 ff. a). Projiziert werden die Orte hier auf die Einteilung der deutschen Dialekte nach Wiesinger (Schmidt, Herrgen \& Kehrein 2008 ff. b). Grau hervorgehoben wird außerdem der Umriss der heutigen Bundesrepublik Deutschland. Dieser ist für die historischen Gegebenheiten natürlich nicht aussagekräftig, erleichtert aber die Orientierung. 


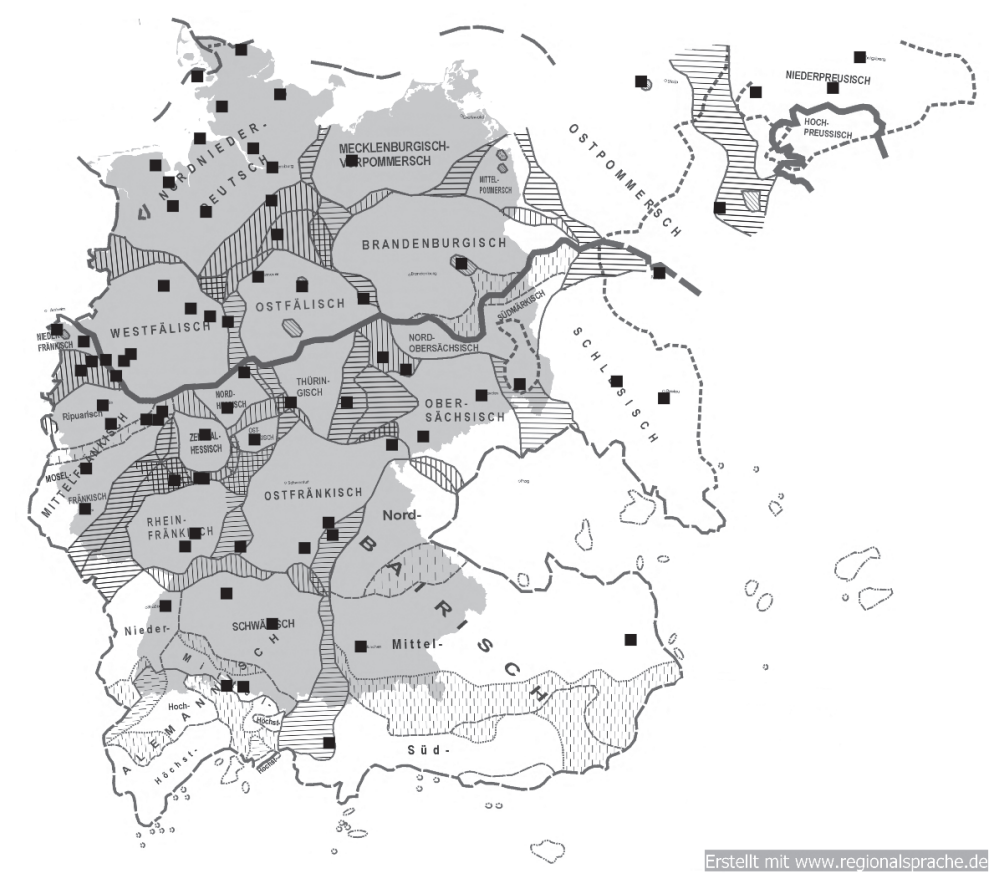

Abbildung 1: Die Herkunft deutschsprachiger ImmigrantInnen

abzugleichen. Da dies über das Erkenntnisinteresse des vorliegenden Aufsatzes hinausgeht (vgl. hierzu aber Zimmer i. Dr.), wird hier eine primär synchrone Analyse der Variation unternommen.

Dass seit dem späten 19. Jahrhundert bzw. dem frühen 20. Jahrhundert auch im Bereich der Kasusmarkierung Sprachwandel in Südwestafrika stattgefunden haben muss, legt die Tatsache nahe, dass wir heute innerhalb des Deutschen in Namibia kaum regionale Unterschiede und vergleichsweise geringe inter-individuelle Variation (vgl. unten sowie Zimmer i. Dr.) und somit eine bemerkenswerte Homogenität beobachten können, die es zu Zeiten der Kolonialisierung sicher noch nicht gegeben hat. Dieser Umstand ist das Resultat von Levelling und Focussing im Varietätenkontakt (vgl. Zimmer i. Dr.). Folglich ist es dann auch nicht nur politisch bzw. geografisch begründet, von einem namibischen Deutschen zu sprechen und somit den Sprachgebrauch aller Deutschsprachigen des Landes zusammenzufassen, sondern auch linguistisch. Damit unterscheidet sich das namibische Deutsche z.B. von Texas German, da es sich bei Letzterem um einen geografisch motivierten Sammelbegriff für zum Teil sehr heterogene Varietäten handelt (vgl. z. B. Boas 2009a: 3). 
Die Bezeichnung Namdeutsch wird schließlich verwendet, um auf einen namibisch geprägten Sprachgebrauch des Deutschen zu referieren, der vom Standarddeutschen abgegrenzt werden kann, das im Land ebenfalls sehr präsent ist. Der Begriff wird auch von der Community mit dieser Bedeutung verwendet und hat meiner Meinung nach deutliche Vorteile gegenüber den (annähernd synonymen) Alternativen Südwesterdeutsch, das vor allem von der jungen Generation wegen der starken Assoziation mit der Kolonialzeit gemieden wird, Nam-Släng, das mit dem stilisierten und von der Community als unauthentisch empfundenen Sprachgebrauch des Rappers EES verknüpft wird und Namlish, das als Kofferwort aus Namibia und English zunächst auf namibisch geprägtes Englisch referierte (vgl. hierzu auch Zimmer 2019: 1185-1187).

Im Folgenden wird nun das Namdeutsche hinsichtlich des Kasusgebrauchs untersucht. Die Grundlage für die Analysen bilden Daten, die während insgesamt drei Feldforschungsreisen in den Jahren 2017 und 2018 erhoben wurden. Herangezogen werden zwei verschiedene Datentypen: Korpusdaten (vgl. Kapitel 3) und Daten, die mithilfe eines Fragebogens im Rahmen einer Nacherhebung gesammelt wurden (vgl. Kapitel 4).

\section{Korpusdaten}

\subsection{Datenerhebung}

Die untersuchten Korpusdaten stammen aus dem Korpus Deutsch in Namibia (DNam; Zimmer et al. 2020). Dieses Korpus enthält Material aus Gesprächsrunden, bei denen sich bis zu fünf Personen in Abwesenheit der ForscherInnen in informellem Setting über Themen klassischer soziolinguistischer Interviews (z. B. Kinderspiele) und/oder alltagsrelevante Aspekte des Lebens in Namibia unterhalten, sowie „Sprachsituationen“ (Bildbeschreibungen in verschiedenen Registern, mündlich und schriftlich) und Interviews (vgl. Zimmer et al. 2020). ${ }^{2}$

2 Die Aufnahmen sind nach den cGAT-minimal-Richtlinien transkribiert. Die Transkripte wurden nach Fertigstellung und Kontrolle semi-automatisch normalisiert und annotiert. Für die Annotation wurde eine für mündliche Kommunikation angepasste Variante des Stuttgart-Tübingen-TagSets (STTS) verwendet (vgl. Westpfahl 2014). Das Korpus ist inzwischen über die Datenbank für gesprochenes Deutsch (DGD) frei zugänglich. Für die Analyse der Kasusverwendung wurde eine noch nicht publizierte Version des Korpus verwendet. Diese Version entspricht im Wesentlichen der finalen Version und beinhaltet alle Transkripte samt Normalisierung und Annotationen. 
Für die vorliegende Studie wurde das Subkorpus untersucht, das die Daten aus den Gesprächsrunden enthält. Hierbei handelt es sich um informelle Gespräche in der Peer-Group oder in der Familie. In aller Regel gingen die Gruppen nach einer kurzen Eingewöhnungsphase in ein flüssiges und lebendiges Gespräch zu selbstgewählten Themen über, sodass wir hier davon ausgehen können, vergleichsweise natürliche Gespräche initiiert und dokumentiert zu haben. Das verwendete Subkorpus hat einen Umfang von 115.004 Tokens und beinhaltet Aufnahmen von 65 SprecherInnen im Alter von 14 bis 65 Jahren (40 Sprecherinnen, 25 Sprecher). Die Gesprächsrunden wurden in den städtischen Zentren aufgenommen, in denen die deutschsprachigen NamibierInnen schwerpunktmäßig leben (Windhoek, Swakopmund, Otjiwarongo und Omaruru) sowie auf Farmen in der Region Omaheke. Insgesamt ist ein breites Spektrum an Bildungsniveaus und Berufen abgedeckt. Einen Schwerpunkt im Korpus stellen Gespräche von bzw. mit SchülerInnen der Geburtsjahrgänge 1999 bis 2003 dar. Komplementiert werden die Korpusdaten durch ein ausführliches Set an SprecherInnen-Metadaten, das mithilfe eines Fragebogens erhoben wurde und bei der quantitativen Auswertung der Gesprächsdaten ebenfalls berücksichtigt wird (s. unten).

Die Korpus-Suchanfrage umfasste alle Artikel und Pronomen in sämtlichen Flexionsformen. Diese wurden mitsamt Kontext und den Metadaten der SprecherInnen extrahiert und manuell von Fehlbelegen bereinigt (z. B. von Belegen mit dem Infinitiv sein, der mit dem Possessivartikel sein homonym ist). Die resultierenden 21.132 Belege wurden anschließend manuell annotiert und mit folgenden Informationen versehen: Wortart (Artikel vs. Pronomen, jeweils mit Subklassifizierung, z. B. Personalpronomen), Kasus des Artikels bzw. des Pronomens (Nominativ, Akkusativ, Dativ, Genitiv oder Sonstiges), Numerus, Genus, Kasuszuweisung (präpositional zugewiesener Kasus?), Belebtheit des Denotats und bei Artikeln außerdem Umfang der Nominalgruppe. Die auf diese Weise gewonnene Datenbasis ermöglicht eine erste fundierte Beschreibung und Analyse der Kasusverwendung im Namdeutschen, die im folgenden Abschnitt vorgenommen wird.

\section{2 Überblick}

Hinsichtlich der Kasusverwendung ist das Namdeutsche - im Gegensatz zum Sprachgebrauch in vielen anderen extraterritorialen deutschsprachigen Communities - als relativ variationsarm zu beschreiben. In weiten Teilen ist die Kasusverwendung dabei deckungsgleich mit dem Standarddeutschen. ${ }^{3}$ So regieren die

3 Wenn ich das Standarddeutsche als Vergleichsvarietät heranziehe, beziehe ich mich dabei auf Paradigmen, wie sie z. B. in der Duden-Grammatik (2016) oder in Eisenberg (2013) zu finden 
Präpositionen durch, für, gegen und um sowie bei dynamischer/direktionaler Verwendung auch die Wechselpräpositionen an, auf, hinter, in, neben, über, unter und $v^{4}{ }^{4}$ in 319 von 324 und somit in 98,5 \% der Fälle im Korpus den Akkusativ (vgl. (2) und (3)). ${ }^{5}$

(2) ich musste immer zeitung kaufn gehn für den schulleiter (NAM117M1)

(3) da habm irgndwelche leute ${ }^{\circ} \mathrm{h}$ ne schnur an das ding gemacht (NAM145M1)

Sogar noch deutlicher ist das Bild bei direkten Objekten (vgl. (4)) und semantischer Kasuszuweisung bei Adverbialen (vgl. (5)). In solchen Fällen steht zu 99,7 \% der Akkusativ (3439 von 3449 Belegen).

(4) aber man man schmeckt den unterschied (NAM171W2)

(5) ich bin busy mit photography jedn bleddie tag (NAM118W1)

Die ausgesprochen wenigen Abweichungen von dieser Systematik (vgl. (6) und (7)) sollten auch in Anbetracht der Tatsache, dass wir es hier mit gesprochensprachlichen Daten zu tun haben, nicht überbewertet werden.

sind. Gesprochensprachliche Varianten wie [nəm] (als mündliches Pendant zu einem), die z. B. in Deppermann, Kleiner \& Knöbl (2013) dokumentiert sind, berücksichtige ich dabei selbstverständlich auch. Eine solche Herangehensweise wirft zwei Probleme auf. Erstens ist auch das Standarddeutsche natürlich nicht frei von morphologischer Variation. Da hier aber die Beschreibung und Analyse des Namdeutschen im Zentrum steht und nicht etwa ein detaillierter Vergleich mit dem Sprachgebrauch in Deutschland, sehe ich davon ab, Regionalstandards oder Ähnliches einzubeziehen. Zweitens wird den Darstellungen in Grammatiken (häufig zurecht) nachgesagt, dass sie traditionell eher an der Schriftsprache orientiert sind als am mündlichen Sprachgebrauch, während hier ja (auch) mündlicher Sprachgebrauch untersucht wird. Das gewählte Vorgehen halte ich dennoch für gerechtfertigt, da gerade in Deutschland das (in Werken wie der Duden-Grammatik beschriebene) Standarddeutsche sowohl in der Schriftlichkeit als auch in der Mündlichkeit als dominante Varietät zu gelten hat (vgl. z. B. Maitz \& Elspaß 2013: 38). Eine weitaus detailliertere Darstellung des Sprachgebrauchs in Deutschland wäre also fraglos möglich, sie erscheint mir im Rahmen dieses Aufsatzes aber nicht zwingend notwendig zu sein.

4 Die Wechselpräposition zwischen ist nur in nicht-direktionaler Verwendung und die Präpositionen bis und ohne sind gar nicht belegt.

5 Bei Beispielen aus unserem Korpus wird im Folgenden immer die jeweilige Sigle des Sprechers bzw. der Sprecherin angegeben, z.B. NAM145M1. Diese beinhaltet die Information, dass der Beleg dem Korpus Deutsch in Namibia entstammt (NAM), eine Nummer, die der Person zugewiesen ist (145), das Geschlecht (M) und eine Angabe zum Geburtsjahr der Person (1: nach 1996; 2: zwischen 1996 und 1977; 3: zwischen 1957 und 1976; 4: vor 1957). 
(6) son mädchen was die nicht mag ich weiß nicht warum ${ }^{\circ} \mathrm{h}$ hat like so etwas in ihr drink gemacht aber das war nich so schlimm (NAM092W1)

(7) die gastgeberin hat ihn dann hat ihm nur drauf hingewiesn hat gesagt ich glaub das is nichts für dich das is wirklich scharf (NAM008W1)

Nominalgruppen und Pronomen in der Funktion des Subjekts sowie mit dem Subjekt kongruierende Nominalgruppen stehen zu 99,9 \% im Nominativ (14.051 von 14.060 Belegen; vgl. (8) und (9)). Auch hier sind Kasusschwankungen demnach zu vernachlässigen.

(8) der ball is immer über die Mauer gegang (NAM088W1)

(9) das is mein ringtone (NAMO30M1)

Selbst vereinzelte Genitiv-Belege finden sich im Korpus (vgl. (10)). Diese sind allerdings die Ausnahme. Stattdessen werden üblicherweise - wie in den meisten anderen Varietäten des Deutschen auch, zumal im mündlichen, informellen Gespräch - Präpositionen und Dativkonstruktionen verwendet (vgl. (11) und (12)).

(10) das war das schlimmste exam meines lebens (NAM117M1)

(11) die oma von meinem freund hat mir welche gemacht (NAM013W1)

(12) zwei operation hatt ich wegn meim knie (NAM090W1)

Interessanter als die bisher thematisierten Strukturen ist aus einer variationslinguistischen Perspektive der Dativ. Hier ist eine ernstzunehmende Schwankung zu beobachten. ${ }^{6}$ Der Dativ (vgl. (13)) konkurriert in erster Linie mit Formen, die mit dem Akkusativ Singular Maskulinum übereinstimmen (vgl. (14)). Diese werden auch bei Neutra verwendet. In solchen Fällen liegt dann eine Form vor, die keine Entsprechung im standarddeutschen Paradigma der Neutra hat (vgl. (15)). Von dieser Schwankung betroffen ist die Kasusmarkierung am Pronomen oder am Artikel. Das substantivische Dativ-Plural- $n$ wird hingegen durchgängig verwendet: In allen 75 Dativ-Plural-Belegen mit Substantiven, die diesen Marker selegieren können (die also kein Plural-s oder Plural-(e)n haben), wird das Flexiv verwendet (vgl. (16)). ${ }^{7}$

6 Für eine Analyse von helfen mit Akkusativ-Komplement im Namdeutschen s. Wiese \& Bracke (i. Ersch.)

7 Abgesehen von diesen 75 Belegen spielen nominale Kasusmarker eine untergeordnete Rolle im Korpus, da Genitive insgesamt äußert selten sind und auch schwache Maskulina nur in geringer Zahl vorkommen. Bei letzteren zeigt sich (wie im Deutschland-Deutschen auch) Variation, da -en in den obliquen Kasus im Singular nicht konsequent verwendet wird: von eim affe gejagt wordn 
(13) das da ist der eine der mit dem ballon immer mal da rüber fliegt (NAM063W1)

(14) is so flippn traurig [...] mit den fuchs (NAM062W1)

(15) da warn wir zwei wieder in den zimmer (NAM090W1)

(16) ja und du findest ja nich wirklich schlang oder so ${ }^{\circ} \mathrm{h}$ weil die sind ja alle weg von den großstädtn (NAM024M1)

Fasst man indirekte Objekte, Wechselpräpositionen in statischer Verwendung und Präpositionen, die kontextunabhängig den Dativ regieren (können), zusammen, kommt man zu einer Anzahl von 1787 Belegen im untersuchten Subkorpus und einem Anteil von 7,1 \% an Formen, die nicht im Dativ stehen (vgl. z. B. (14) und (15)). Auch wenn die Dativverwendung hier deutlich überwiegt, haben wir es dennoch mit einer ernstzunehmenden und nicht marginalen Schwankung zu tun, die im Folgenden eingehender untersucht werden soll.

Vergleichbare Schwankungsfälle lassen sich in beinahe allen anderen extraterritorialen Varietäten des Deutschen beobachten, wo ebenfalls besonders die Dativmarkierung von Variation und Wandel betroffen ist (s. Kapitel 5). Bei deren Untersuchung hat sich immer wieder gezeigt, dass eine Vielzahl miteinander interagierender Faktoren bei der Beschreibung und Erklärung des Phänomens berücksichtigt werden muss (vgl. z.B. Salmons 1994: 68). Wie weiter unten ersichtlich wird, ist auch im Namdeutschen ein komplexes Geflecht an Faktoren relevant. Um diesem Umstand gerecht zu werden, wird die Dativverwendung im Namdeutschen deshalb mithilfe eines multifaktoriellen statistischen Modells untersucht. Auf diese Weise lässt sich die Bedeutung von einzelnen grammatischen Merkmalen und soziobiographischen Eigenschaften der SprecherInnen ermessen. Außerdem können Strukturen identifiziert werden, die besonders progressiv oder besonders konservativ verwendet werden.

\subsection{Analyse}

In diesem Abschnitt soll untersucht werden, welche Faktoren einen Einfluss darauf haben, ob die SprecherInnen in den von uns aufgezeichneten und transkribierten Gesprächen einen Dativ verwenden oder nicht. Die abhängige Variable ist demnach KASUS mit den binären Merkmalsausprägungen Dativ und kein_Dativ. Analysiert wird die Kasusmarkierung durch Artikel und Pronomen in Kontexten, in denen im Korpus mindestens einmal ein Dativ verwendet wird: bei indirekten

(NAM019M1) vs. lass den namen da (NAM063W1). Weiterführende Aussagen sind aufgrund der niedrigen Belegzahl nicht möglich. 
Objekten und in Präpositionalphrasen mit den statisch verwendeten Wechselpräpositionen an, auf, hinter, in, neben, über, unter, vor, zwischen und den Präpositionen aus, bei, mit, nach, von, wegen, zu. Getestet wird der potentielle Einfluss von Faktoren, die sich auf grammatische Merkmale der untersuchten Struktur beziehen, sowie von Faktoren, die sich aus soziobiographischen Eigenschaften der SprecherInnen ableiten lassen.

Da eine binäre abhängige Variable vorliegt, der Einfluss mehrerer Faktoren untersucht werden soll und pro SprecherIn mehrere Beobachtungen (also zu analysierende Äußerungen) vorliegen, wurde für die Analyse der Daten ein binomial generalised linear mixed model (GLMM) gewählt (s. hierzu z. B. Baayen 2008: 278284). Dazu wurde die Software $R$ verwendet (R Core Team 2019) und das Package lme4 (Bates et al. 2015). Zunächst wurde ein maximales Modell berechnet, das alle unabhängigen Variablen enthält, deren Einfluss auf die Ausprägung der abhängigen Variable getestet werden soll. Darüber hinaus wurde SPRECHERIN als random effect integriert. Auf diese Weise wird sichergestellt, dass möglicherweise $\mathrm{zu}$ beobachtendes idiosynkratrisches Verhalten einzelner SprecherInnen nicht zu stark ins Gewicht fällt. Das maximale Modell ist in (17) dokumentiert. Die einzelnen Variablen werden weiter unten erläutert.

(17) Kasus $\sim$ Wortart + Numerus + Genus + Kasuszuweisung + Semantik + Alter + Geburtsland + Geschlecht + Schule + M_L1_Deutsch + V_L1_Deutsch + Kontakt_Deutschlanddeutsche + (1|SprecherIn)

Dieses maximale Modell enthält nun unabhängige Variablen, die die Modellgüte verbessern und einen signifikanten Einfluss auf die Ausprägung der abhängigen Variable haben und solche, bei denen dies nicht der Fall ist. Um Letztere aus dem Modell zu entfernen, wurden verschiedene Modellvarianten miteinander verglichen, bei denen jeweils eine Variable entfernt wurde, sodass ersichtlich wird, welche Variablen die Modellgüte verbessern und welche nicht (vgl. z. B. Levshina 2015: 266-267). Das Ergebnis ist in Tabelle 1 abgebildet. Entscheidend ist hier, welche Variablen das Signifikanzniveau von 0.05 unterschreiten (zweite Spalte von rechts), was durch Asteriske in der Spalte ganz rechts veranschaulicht wird. Drei Asteriske stehen für einen höchstsignifikanten $(\mathrm{p}<0.001)$, zwei Asteriske für einen hochsignifikanten $(p<0.01)$ und ein Asterisk für einen signifikanten Einfluss ( $\mathrm{p}<0.05)$. Ein Punkt bedeutet, dass das veranschlagte Signifikanzniveau nur knapp nicht erreicht wurde $(0.05<\mathrm{p}<0.1)$. 
Tabelle 1: Überblick über den Einfluss der unabhängigenVariablen

\begin{tabular}{llllll}
\hline Variable & Df & AIC & LRT & $\operatorname{Pr}($ Chi) & \\
\hline WORTART & 5 & 665.55 & 31.328 & $8.07 \mathrm{E}-06$ & $\star \star \star$ \\
NUMERUS & 1 & 666.82 & 24.603 & $7.05 \mathrm{E}-07$ & $\star \star \star$ \\
GENUS & 3 & 675.9 & 37.683 & $3.30 \mathrm{E}-08$ & $\star \star \star$ \\
KASUSZUWEISUNG & 2 & 655.7 & 15.481 & 0.0004348 & $* \star \star$ \\
SEMANTIK & 1 & 642.39 & 0.169 & 0.6809236 & \\
ALTER & 2 & 642.71 & 2.49 & 0.2879675 & \\
GEBURTSLAND & 2 & 641.02 & 0.795 & 0.6718819 & \\
GESCHLECHT & 1 & 642.22 & 0 & 1.0000000 & \\
SCHULE & 6 & 649.3 & 17.081 & 0.0089887 & $\star \star$ \\
M_L1_DEUTSCH & 1 & 654.13 & 11.91 & 0.0005584 & $* \star *$ \\
V_L1_DEUTSCH & 1 & 642.33 & 0.106 & 0.744931 & \\
KONTAKT_DEUTSCHLANDDEUTSCHE & 1 & 645.38 & 3.156 & 0.0756442 &. \\
\hline
\end{tabular}

Keinen signifikanten Einfluss haben demnach die soziolinguistischen Variablen Alter, Geburtsland, Geschlecht, V_L1_Deutsch und Kontakt_DeutschLANDDEUTSCHE. Die Variable GeBuRTSLAND differenziert zwischen Mitgliedern der Community, die in Namibia geboren wurden und solchen, die in Deutschland oder Südafrika geboren wurden. Da es hier keinen signifikanten Effekt gibt, scheinen sich zugewanderte NamibierInnen hinsichtlich des Kasusgebrauchs sprachlich nicht vom Rest der Community abzusetzen (was nicht verwundert, da viele der im Ausland geborenen SprecherInnen, deren Sprachgebrauch im Korpus dokumentiert ist, bereits seit Langem Teil der Community sind). Die Variable V_L1_Deutsch bezieht sich auf Angaben zu den Sprachen der Eltern, die mitsamt den Metadaten erhoben wurden. Die zugrundeliegende Frage ist in (18) abgebildet.

(18) Welche Sprache(n) sprechen/sprachen Deine Eltern? (bitte sortieren und mit der am besten gesprochenen Sprache beginnen)

Mutter:

Vater:

Die Antworten wurden dergestalt in die Variablen M_L1_DEuTSCH und V_L1_ Deutsch überführt, dass der Wert ja vergeben wurde, wenn Deutsch als Erstsprache genannt wurde und nein, wenn eine andere Sprache zuerst genannt wurde. M_L1_Deutsch bezieht sich auf die Mutter der jeweiligen SprecherIn, V_L1_DEuTsCH auf den Vater. Diese Kategorisierung ist natürlich nicht unproblematisch, da so nicht angegeben werden kann, wenn Elternteile zwei gleichberechtigte Erstsprachen haben. In aller Regel bereitete die Frage nach der Reihung der Sprachen den TeilnehmerInnen aber keine Schwierigkeiten, da diesbezüglich 
bei vielen TeilnehmerInnen offenbar keine Unsicherheiten herrschen. Darüber hinaus gehen durch die Überführung in eine binäre Variable natürlich Informationen verloren, z. B. darüber, ob Deutsch überhaupt vom Elternteil gesprochen wird. Hier bieten sich für die Zukunft genauere und qualitative Analysen an, die diesen Aspekt genauer in den Blick nehmen. Für die quantitative Auswertung, die im Zentrum des vorliegenden Aufsatzes steht, musste allerdings eine praktikable Operationalisierung gefunden werden.

Dass nur eine der beiden Variablen einen signifikanten Einfluss hat, erscheint mir besonders aufschlussreich: Lediglich die Erstsprache der Mutter hat einen Einfluss auf den Kasusgebrauch im Korpus (weitere Details s. unten). Ist die Erstsprache der Mutter nicht Deutsch, verwenden die Kinder seltener Dativformen und weichen häufiger vom standarddeutschen Muster ab. Die Erstsprache des Vaters hat hingegen keinen signifikanten Einfluss auf den Dativgebrauch der Nachfahren. Möglicherweise spiegelt sich hier die Rollenverteilung innerhalb der Community: Es sind vor allem die Mütter, die sich um die Erziehung kümmern und auf diese Weise offenbar einen größeren Einfluss auf den Sprachgebrauch der Kinder haben.

Neben V_L1_Deutsch hat auch KonTAKT_DeutschlandDEUTsche keinen signifikanten Einfluss. Diese Variable berücksichtigt, wie häufig die jeweilige Person Kontakt mit Deutschlanddeutschen hat (von nie über ein Mal im Jahr, ein Mal im Monat und ein Mal in der Woche bis hin zu öfter). Ebenso ohne signifikanten Einfluss ist schließlich die Variable SEMANTIK. Da in der Literatur zum Kasusgebrauch in deutschen Sprachinseln immer wieder die Bedeutung der Belebtheitshierarchie betont wird (s. unten), wurde hier hinsichtlich des semantischen Merkmals [+/- menschlich] differenziert. Für eine Unterscheidung zwischen [+belebt] und [-belebt] bei Denotaten, die das Merkmal [-menschlich] haben, lagen nicht genügend Belege mit der Merkmalskombination [+belebt] und [-menschlich] vor. Siehe hierzu aber Kapitel 4.

Die Variablen ohne signifikanten Einfluss wurden in einem zweiten schlankeren Modell eliminiert. Die entsprechende Modell-Spezifikation ist in (19) dargestellt.

(19) Kasus $\sim$ Wortart + Numerus + Genus + Kasuszuweisung + Schule + M_L1_Deutsch + (1|SprecherIn)

Zur Beschreibung der Modellgüte seien die folgenden Werte genannt: der marginal- $\mathrm{R}^{2}$ Wert liegt bei 0.2335678 , conditional- $\mathrm{R}^{2}$ (der im Gegensatz zum erstgenannten Wert auch den random effect SprecherIn berücksichtigt) bei 0.2696258. Der index of concordance (C), der bemisst, wie gut ein Modell dazu in der Lage ist, die Ausprägung der abhängigen Variable vorherzusagen, hat einen Wert von 
0.9091417 (zu diesem Wert vgl. Levshina 2015: 259, Baayen 2008: 204). Werte von C $\geq 0.9$ klassifizieren Hosmer \& Lemeshow (2000: 162) als „outstanding discrimination“. Allerdings sollten hier auch die Werte der Konfusionstabelle berücksichtigt werden (vgl. Tabelle 2).

Tabelle 2: Konfusionstabelle

\begin{tabular}{cccc}
\hline \multirow{2}{*}{ Vorhergesagt } & \multicolumn{2}{c}{ Beobachtet } & $\begin{array}{c}\text { Anteil korrekter } \\
\text { Vorhersagen }\end{array}$ \\
\cline { 2 - 3 } Dativ & Dativ & kein_Dativ & \\
kein_Dativ & 1607 & 98 & $94,3 \%$ \\
& 8 & 29 & $78,4 \%$ \\
\hline
\end{tabular}

Diesem Überblick ist zu entnehmen, dass insgesamt 93,4\% aller Merkmalsausprägungen der abhängigen Variable korrekt vorhergesagt werden (bei 98 false positives und 8 false negatives). Dieser Wert liegt nur knapp, aber signifikant über dem Anteil der häufigsten Merkmalsausprägung der abhängigen Variable (ein Dativ liegt in 92,7\% der Beobachtungen vor; $\mathrm{p}=0.02705^{\star}$ ).

Weiterhin liegt keine Multikollinearität vor. Es kann also nicht von einer Merkmalsausprägung der abhängigen Variable auf die Merkmalsausprägung einer anderen Variable geschlossen werden, was die Aussagekraft der Koeffizienten schmälern würde (vgl. z. B. Backhaus et al. 2003: 88-91): Die Variation Inflation Factors (VIF) übersteigen den Wert von 3 nicht und sind somit unproblematisch (vgl. z. B. Levshina 2015: 272).

Tabelle 3 enthält die Ergebnisse des Modells. Pro Variable ist dargestellt, welchen Einfluss eine Merkmalsausprägung (z. B. Demonstrativpronomen) auf die Ausprägung der abhängigen Variable hat (Dativ vs. kein_Dativ), und zwar im Kontrast zum Referenzlevel der Variable (z. B. Definitartikel). Wichtig sind auch hier der p-Wert und seine Veranschaulichung in den beiden Spalten ganz rechts. Für die Interpretation der Ergebnisse ebenfalls wichtig sind die Estimates in der zweiten Spalte von links. Diese geben an, in welche Richtung sich ein Effekt auswirkt: Ein positiver Wert bedeutet, dass das Vorhandensein der jeweiligen Merkmalsausprägung die Wahrscheinlichkeit von kein_Dativ erhöht. Je stärker der Wert von 0 abweicht, desto stärker ist der Effekt. Demnach gibt es z. B. keinen signifikanten Unterschied hinsichtlich der Wahrscheinlichkeit, dass kein_Dativ auftritt zwischen den Merkmalsausprägungen Definitartikel und Demonstrativpronomen (da p > 0.05), wohl aber zwischen Definitartikel und Indefinitartikel: Ein Indefinitartikel erhöht die Wahrscheinlichkeit, dass kein_Dativ zu beobachten ist. 
Tabelle 3: Ergebnisse der GLMM

\begin{tabular}{|c|c|c|c|c|c|}
\hline & Estimate & Std. Error & $z$ value & $\operatorname{Pr}(>|z|)$ & \\
\hline (Intercept) & -6.781051 & 0.68842 & -9.85 & $<2 \mathrm{e}-16$ & $\star \star \star *$ \\
\hline WORTART & \multicolumn{5}{|c|}{ Referenzlevel: Definitartikel } \\
\hline Demonstrativpronomen & -0.260308 & 0.474079 & -0.549 & 0.582949 & \\
\hline Indefinitartikel & 0.891787 & 0.318712 & 2.798 & 0.005141 & $\star \star$ \\
\hline Personalpronomen & -2.378641 & 0.814233 & -2.921 & 0.003485 & $\star \star$ \\
\hline Possessivartikel & 0.797945 & 0.302527 & 2.638 & 0.00835 & $\star \star$ \\
\hline sonstige & -0.419075 & 0.477093 & -0.878 & 0.37973 & \\
\hline NUMERUS & \multicolumn{5}{|c|}{ Referenzlevel: Plural } \\
\hline Singular & 1.903418 & 0.512975 & 3.711 & 0.000207 & $\star \star *$ \\
\hline Genus & \multicolumn{5}{|c|}{ Referenzlevel: Femininum } \\
\hline kein_Genus & 0.009589 & 0.77767 & 0.012 & 0.990162 & \\
\hline Maskulinum & 1.249104 & 0.289093 & 4.321 & $1.55 \mathrm{E}-05$ & $\star \star \star$ \\
\hline Neutrum & 1.750717 & 0.311371 & 5.623 & $1.88 \mathrm{E}-08$ & $\star \star \star$ \\
\hline KASUSZUWEISUNG & \multicolumn{5}{|c|}{ Referenzlevel: Wechselpraeposition } \\
\hline nicht_praep & 1.454348 & 0.356968 & 4.074 & $4.62 \mathrm{E}-05$ & $\star * *$ \\
\hline Praeposition & 0.350513 & 0.27022 & 1.297 & 0.194584 & \\
\hline SCHULE & \multicolumn{5}{|c|}{ Referenzlevel: DHPS } \\
\hline Delta & 1.411645 & 0.559622 & 2.522 & 0.011653 & * \\
\hline englischsprachig & 1.08051 & 0.566845 & 1.906 & 0.056626 & . \\
\hline Namib_High & 2.508723 & 0.581191 & 4.317 & $1.59 \mathrm{E}-05$ & $\star \star \star$ \\
\hline oss & 0.564514 & 0.56661 & 0.996 & 0.319104 & \\
\hline PSS & 1.593544 & 0.519115 & 3.07 & 0.002142 & $\star \star$ \\
\hline sonstige & 0.340574 & 0.623664 & 0.546 & 0.585007 & \\
\hline M_L1_DEUTSCH & \multicolumn{5}{|c|}{ Referenzlevel: ja } \\
\hline nein & 1.702314 & 0.484084 & 3.517 & 0.000437 & $\star \star \star$ \\
\hline
\end{tabular}

Im Folgenden werden die Variablen mit signifikantem Einfluss kurz erläutert. Interpretiert werden die Ergebnisse in Kapitel 5, nachdem die Fragebogendaten, die als zweiter Datentyp herangezogen werden, erläutert und analysiert wurden (vgl. Kapitel 4), sodass die abschließende Auswertung der Ergebnisse beide Datentypen gleichermaßen aufgreifen kann.

Zunächst zur WoRTART: Hier wird differenziert zwischen Demonstrativpronomen (z.B. was ich an dem gut finde ist [...], NAM019M1), Personalpronomen (ich hatte das davor schon mal bei ihm geübt, NAM024M1), Definitartikel (deine 
soße die wir hattn bei dem basar [...], NAM008W1) ${ }^{8}$, Indefinitartikel (und bei eim strau $\beta$ muss du dich hinlegn, NAM138W1), Possessivartikel (was macht der in deim Busch, NAM023W1) und sonstigem Artikel/Pronomen. ${ }^{9}$

NumERUS unterscheidet zwischen Singular- und Pluralformen. Auch GENUS bezieht sich auf eine grammatische Kategorie. Die Merkmalsausprägung kein_ Genus wird benötigt für bestimmte Pluralformen (z.B. Eltern) sowie Pronomen (z.B. uns). Die Variable KASUSZUWEISUNG differenziert zwischen Wechsel- und sonstigen Präpositionen sowie indirekten Objekten. Und schließlich werden die Schulen, die die SprecherInnen besuch(t)en, durch die entsprechende Variable abgedeckt. Delta und DHPS sind Windhoeker Schulen, Namib High und PSS sind in Swakopmund und die OSS in Otjiwarongo beheimatet. englischsprachig fasst mehrere Windhoeker Schulen zusammen, die DaM nicht anbieten (die im Korpus vertretenen SchülerInnen dieser Schulen erhalten aber privaten DaM-Unterricht).

\section{Fragebogendaten}

Ergänzend zu den Korpusdaten wird im Folgenden auch eine Fragebogenstudie ausgewertet. Hierbei handelt es sich um Daten, die im Rahmen einer Nacherhebungsphase und auf den Erkenntnissen erster Korpsauswertungen aufbauend gezielt erhoben wurden, um konkrete Fragestellungen bearbeiten zu können. So wird z. B. bei der Erklärung von Kasusschwankungen in Sprachinseln immer wieder die Bedeutung der Belebtheitshierarchie betont (vgl. z. B. Salmons 1994, Yager et al. 2015 und Rosenberg 2016). Deren Relevanz kann aufgrund zu weniger Belege für beispielsweise belebte, aber nicht menschliche Denotate in einem obliquen Kasus anhand unserer Korpusdaten aber nicht präzise eruiert werden. Um dennoch Aussagen zu diesem Aspekt machen zu können, wurden entsprechende Items in unsere Nacherhebungsstudie aufgenommen. Außerdem ermöglicht die Analyse eines zweiten Datentyps, die Verlässlichkeit der Ergebnisse zu

8 Bei Wortformen von der/die/das wird mitunter eine Unterscheidung zwischen rein phorischer und ana- bzw. katadeiktischer Funktion vorgenommen (der/die/das als Definitartikel vs. Demonstrativartikel; Duden-Grammatik 2016: 280). Diese in der Literatur umstrittene Unterscheidung (vgl. Thieroff \& Vogel 2012: 70-71 und Eisenberg 2013: 157-158), die vor allem auf der Betonbarkeit des Artikels basiert, wird hier nicht vorgenommen. Als Demonstrativartikel werden hier demnach nur solche Wortformen klassifiziert, die sich auf formaler Ebene in mehr als der (oft schwer zu bestimmenden) Betonbarkeit von Definitartikeln unterscheiden (also z. B. diesem). 9 Zur letzten Kategorie zählen die allesamt äußerst infrequenten Demonstrativartikel (z.B. diesem), Indefinitpronomen (z. B. jemandem), Possessivpronomen (z. B. meinem), QuantifikativPronomen (allem), Quantifikativ-Artikel (jedem), Interrogativ-Pronomen (z. B. wem) und Interrogativ-Artikel (welchem). 
testen, indem überprüft wird, ob eine andere Methode zu vergleichbaren Resultaten führt. Dies schließt hier auch ein anderes Medium ein: Während bisher ausschließlich mündlicher Sprachgebrauch untersucht wurde, liefert die Fragebogenstudie handschriftliche Daten, was insofern günstig ist, als hier geprüft werden kann, ob phonologische Abweichungen von Dativ-Formen sich auch in schriftlichen Daten widerspiegeln.

\subsection{Datenerhebung}

Um gezielt Dativformen bzw. deren Alternativen zu elizitieren, wurde eine Fragebogenstudie im Lückentext-Design durchgeführt. Die Items waren so konzipiert, dass jeweils ein Satzanfang weitergeführt werden sollte, sodass ein vollständiger Satz entsteht. Bei der Vervollständigung mussten bestimmte vorgegebene Lexeme verwendet werden (ein bis drei Lexeme pro Item). Die konkrete Anweisung ist in (20) abgedruckt.

(20) Bitte vervollständige die Satzanfänge so, dass ein vollständiger Satz entsteht. Ergänze die Satzanfänge spontan und denk nicht zu viel darüber nach. Es sollen Sätze entstehen, die für dich persönlich gebräuchlich sind. Namdeutsch/Namlish/Namsläng/ Südwesterdeutsch ist erlaubt! Wichtig: Die Wörter in der rechten Spalte müssen verwendet werden. Sie dürfen abgeändert werden und es dürfen weitere Wörter verwendet werden.

Mit jeder Gruppe wurde zunächst eine Testphase durchgeführt, damit sich die TeilnehmerInnen mit dem Design vertraut machen konnten und um sicherzugehen, dass die Anweisung verstanden wurde. Die Satzanfänge wurden von einem Mitglied der deutschsprachigen Community in Namibia eingesprochen und bei der Datenerhebung auditiv präsentiert. Die Vervollständigung der Sätze durch die TeilnehmerInnen erfolgte dann handschriftlich. Auf dem Fragebogen waren neben der Satz-Nummer nur die zu verwendenden Lexeme bzw. das zu verwendende Lexem abgedruckt. Der Satzanfang wurde nicht schriftlich dargeboten (vgl. das Beispiel in (21)).

(21) Satz Nr. 2

| Hund

Das Ausfüllen der Lücken erfolgte mit einer konkreten Zeitvorgabe, die bewusst knapp gehalten wurde, damit die TeilnehmerInnen möglichst spontan reagieren und keine Zeit haben, die Texte metasprachlich zu reflektieren. Alle Items wurden mithilfe einer Playlist unmittelbar nacheinander abgespielt mit jeweils 
einem Zeitraum von 10 Sekunden für das Ausfüllen der Lücken. Das Ende dieser Zeiträume wurde jeweils mit einem akustischen Signal nach 8 Sekunden angekündigt. Insgesamt waren 34 Satzanfänge $\mathrm{zu}$ vervollständigen. Da immer nur wenige Wörter ergänzt werden mussten, waren alle TeilnehmerInnen gut dazu in der Lage, diese Aufgabe zu erfüllen. Nach 17 Items wurde mit allen Gruppen eine kurze Pause eingelegt.

Von den 34 Items sind elf dazu konzipiert, Dativformen bzw. deren Alternativen zu elizitieren (vgl. Tabelle 4). Bei den anderen 24 Items handelt es sich um Filler bzw. Items, die für die Untersuchung anderer Phänomene benötigt werden. Um Reihenfolge-Effekten vorzubeugen, wurden die Items den verschiedenen Gruppen in zwei unterschiedlichen (jeweils randomisierten) Abfolgen präsentiert.

Tabelle 4: Lückentext-Items zur Elizitation von Dativformen bzw. deren Alternativen

\begin{tabular}{|c|c|c|c|c|}
\hline & Stimulus & $\begin{array}{l}\text { zu } \\
\text { ergänzendes } \\
\text { Lexem }\end{array}$ & Semantik & Genus \\
\hline 1 & $\begin{array}{l}\text { Ich beobachte eine schöne schwarze Katze. } \\
\text { Dann kommt ein Junge und spielt mit ... }\end{array}$ & Katze & belebt & $f$ \\
\hline 2 & $\begin{array}{l}\text { Ein Hund sitzt im Wohnzimmer. Schnell kommen } \\
\text { zwei Kinder und spielen mit ... }\end{array}$ & Hund & belebt & $\mathrm{m}$ \\
\hline 3 & $\begin{array}{l}\text { Am Denkmal standen einige Touristen. Ich ging } \\
\text { hin und sprach mit ... }\end{array}$ & Frau & menschlich & $f$ \\
\hline 4 & $\begin{array}{l}\text { Eine Frau ist allein im Park. Dann kommt ein } \\
\text { nettes Mädchen und spricht ... }\end{array}$ & mit & menschlich & $f$ \\
\hline 5 & $\begin{array}{l}\text { Erst standen mein Onkel und eine Frau still } \\
\text { nebeneinander. Doch dann redete mein Onkel } \\
\text { mit ... }\end{array}$ & Frau & menschlich & $f$ \\
\hline 6 & $\begin{array}{l}\text { Ein Mann geht über eine Straße. Auf einmal } \\
\text { kommt ein Polizist und spricht ... }\end{array}$ & mit & menschlich & $\mathrm{m}$ \\
\hline 7 & $\begin{array}{l}\text { Ein Mann und eine Frau streiten. Er schreit aber } \\
\text { sie redet ganz normal mit ... }\end{array}$ & Mann & menschlich & $\mathrm{m}$ \\
\hline 8 & $\begin{array}{l}\text { Ich kannte niemanden auf der Party. Am Ende } \\
\text { habe ich trotzdem mit ... }\end{array}$ & $\begin{array}{l}\text { Mann; } \\
\text { sprechen }\end{array}$ & menschlich & $\mathrm{m}$ \\
\hline 9 & $\begin{array}{l}\text { Ein Dieb möchte eine kleine Tasche stehlen. Ein } \\
\text { Mann wehrt sich und schlägt den Dieb mit ... }\end{array}$ & Tasche & unbelebt & $f$ \\
\hline 10 & $\begin{array}{l}\text { Zwei Kinder spielen miteinander. Das Mädchen } \\
\text { hat ein Kissen in der Hand. Plötzlich holt das } \\
\text { Mädchen aus und schlägt den Jungen mit ... }\end{array}$ & Kissen & unbelebt & $\mathrm{n}$ \\
\hline 11 & $\begin{array}{l}\text { Das Einkaufszentrum ist weit weg. Wir laufen } \\
\text { nicht, sondern fahren ... }\end{array}$ & Auto & unbelebt & $n$ \\
\hline
\end{tabular}


Um Aufschluss über die Relevanz der Belebtheit geben zu können, variieren die Items hinsichtlich des zu ergänzenden Lexems. Darüber hinaus werden verschiedene Genera abgedeckt. Um sowohl Artikel als auch Pronomen elizitieren zu können, sind nicht nur Substantive vorgegeben, sondern manchmal auch nur die Präposition mit (s. Zeile 4 und 6 in Tabelle 4). Hier wurden zusätzlich zur Präposition mitunter auch volle Nominalgruppen ergänzt, in der Regel aber Pronomen. Konstant gehalten wurde die Kasuszuweisung über die Präposition mit sowie der Numerus (Singular). Auf diese Weise reduziert sich die Anzahl der möglichen grammatischen Prädiktoren für die Analyse. Auch die Zahl der soziolinguistischen Variablen ist geringer, da bei der Fragebogenerhebung ein im Vergleich zum Korpus wesentlich kleineres Set an (sprach-)biographischen Informationen abgefragt wurde, sodass mehr Zeit für Elizitationen und andere Forschungsdesigns aufgewendet werden konnte (z. B. wurden die TeilnehmerInnen nicht gefragt, welche Schule sie besucht haben, sodass die entsprechende Variable in der Analyse unten entfällt).

An der Fragebogenstudie nahmen 184 NamibierInnen teil und somit knapp $1 \%$ der deutschsprachigen Community im Land (72 weibliche und 108 männliche TeilnehmerInnen sowie vier Personen, die keine Angabe zum Geschlecht gemacht haben). Die jüngsten TeilnehmerInnen waren 14, die älteste Teilnehmerin 67 Jahre alt. Die Daten wurden in Windhoek, Swakopmund und Otjiwarongo erhoben. Die Mehrzahl der Bögen wurde von SchülerInnen der dortigen Schulen ausgefüllt. In jedem Erhebungsort konnte aber auch eine signifikante Anzahl an Erwachsenen akquiriert werden.

\subsection{Analyse}

Wie im Korpus überwiegen die Dativformen auch in der Fragebogenstudie: In 93,9 \% der Fälle wählten die ProbandInnen eine solche Form. Dieser Wert liegt erstaunlich nah am Anteil der Dativformen im Korpus: Bei den relevanten Präpositionen wurde dort zu 92,8 \% ein Dativ verwendet und nach der Präposition mit zu $91 \%$.

Um Aufschluss über die Faktoren zu geben, die die Kasuswahl im Lückentext steuern, wurde auch für diesen Datentyp eine GLMM berechnet. Die Vorgehensweise entspricht der in Kapitel 3, weshalb hier die relevanten Informationen ohne eine detaillierte Erläuterung der Vorgehensweise geliefert werden. In (22) ist die Spezifikation für das maximale Modell gegeben.

(22) Kasus $\sim$ Wortart + Genus + Semantik + Altersgruppe + Geschlecht + Geburtsland + M_L1_Deutsch + V_L1_Deutsch + (1|SprecherIn) + (1|Item) 
Das in (23) dargestellte Modell ergibt sich aus der Eliminierung der Variablen ohne signifikanten Einfluss (vgl. Tabelle 5).

Tabelle 5: Überblick über den Einfluss der unabhängigen Variablen

\begin{tabular}{llllll}
\hline Variable & Df & AIC & LRT & $\operatorname{Pr}($ Chi) & \\
\hline WORTART & 3 & 590.03 & 11.1695 & 0.010844 & $*$ \\
GENUS & 2 & 594.18 & 13.319 & 0.001282 & $\star \star$ \\
SEMANTIK & 2 & 582.28 & 1.4205 & 0.491527 & \\
ALTER & 1 & 583.14 & 0.2838 & 0.594238 & \\
GESCHLECHT & 1 & 598.52 & 15.6588 & $7.59 \mathrm{E}-05$ & $* \star$ \\
GEBURTSLAND & 2 & 581.18 & 0.3188 & 0.852641 & \\
M_L1_DEUTSCH & 1 & 589.17 & 6.31 & 0.012006 & $*$ \\
V_L1_DEUTSCH & 1 & 589.96 & 7.1042 & 0.00769 & $*$ \\
\hline
\end{tabular}

(23) Kasus $\sim$ Wortart + Genus + Geschlecht + M_L1_Deutsch + V_L1_Deutsch + (1|SprecherIn) + (1|Item)

Die Modellgüte lässt sich an der Konfusionstabelle (vgl. Tabelle 6) festmachen sowie an folgenden Werten: $\mathrm{R}^{2}$ (marginal): 0.01705465, $\mathrm{R}^{2}$ (conditional): 0.04587572, C: 0.9657049. Die $\mathrm{R}^{2}$-Werte sind als niedrig einzustufen (was bedeutet, dass nur ein geringer Teil der Variation erklärt werden kann), während C und die Konfusionstabelle dafür sprechen, dass die Ausprägung der abhängigen Variable gut vorhergesagt werden kann. Wie bei den Korpusdaten liegt der Anteil der korrekt vorhergesagten Werte knapp, aber signifikant über dem Anteil der häufigsten Merkmalsausprägung der abhängigen Variable $\left(\mathrm{p}<0.001^{\star \star \star}\right)$. Multikollinearität liegt nicht vor, da die VIFs den Wert von 2 nicht übersteigen.

Tabelle 6: Konfusionstabelle

\begin{tabular}{cccc}
\hline \multirow{2}{*}{ Vorhergesagt } & \multicolumn{2}{c}{ Beobachtet } & $\begin{array}{c}\text { Anteil korrekter } \\
\text { Vorhersagen }\end{array}$ \\
\cline { 2 - 3 } Dativ & Dativ & kein_Dativ & \\
kein_Dativ & 1681 & 67 & $96,2 \%$ \\
& 11 & 43 & $79,6 \%$ \\
\hline
\end{tabular}

Tabelle 7 gibt schließlich Aufschluss über den Einfluss der einzelnen Merkmalsausprägungen. 
Tabelle 7: Ergebnisse der GLMM

\begin{tabular}{|c|c|c|c|c|c|}
\hline & Estimate Std. & Error $\mathrm{z}$ & value & $\operatorname{Pr}(>|z|)$ & \\
\hline (Intercept) & -4.2831 & 0.6047 & -7.083 & $1.41 \mathrm{E}-12$ & $\star \star \star *$ \\
\hline WORTART & \multicolumn{5}{|c|}{ Referenzlevel: Indefinitartikel } \\
\hline Definitartikel & -0.7788 & 0.3387 & -2.3 & 0.02147 & * \\
\hline Personalpronomen & -1.604 & 0.5159 & -3.109 & 0.00187 & $\star \star$ \\
\hline sonstige & -0.3173 & 0.6949 & -0.457 & 0.64797 & \\
\hline Genus & \multicolumn{5}{|c|}{ Referenzlevel: Femininum } \\
\hline Maskulinum & 1.3242 & 0.3009 & 4.401 & $1.08 \mathrm{E}-05$ & $\star \star \star \star ~$ \\
\hline Neutrum & 0.7815 & 0.3818 & 2.047 & 0.04068 & * \\
\hline GESCHLECHT & \multicolumn{5}{|c|}{ Referenzlevel: $\mathrm{m}$} \\
\hline w & -2.4863 & 0.5663 & -4.39 & $1.13 \mathrm{E}-05$ & 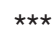 \\
\hline M_L1_DEUTSCH & \multicolumn{5}{|c|}{ Referenzlevel: ja } \\
\hline nein & 1.5002 & 0.6432 & 2.332 & 0.01968 & * \\
\hline V_L1_DEUTSCH & \multicolumn{5}{|c|}{ Referenzlevel: ja } \\
\hline nein & 1.8305 & 0.5969 & 3.067 & 0.00216 & $\star \star$ \\
\hline
\end{tabular}

\section{Interpretation der Ergebnisse}

Im Folgenden werden die Ergebnisse der beiden vorangehenden Kapitel interpretiert. Dazu werden zunächst die beiden unterschiedlichen Datentypen miteinander verglichen, bevor der Fokus in den sich anschließenden Abschnitten zunächst auf den soziolinguistischen (Abschnitt 5.2) und dann auf den grammatischen Variablen (5.3) liegt.

\subsection{Vergleich der Datentypen}

Beim Vergleich der beiden herangezogenen Datentypen fällt zunächst einmal ins Auge, dass die jeweiligen Analyseergebnisse nahezu deckungsgleich sind. Das betrifft zum einen den Anteil der Dativformen (s. oben). Die hier festzustellende Übereinstimmung der Ergebnisse spricht für deren Verlässlichkeit. Zum anderen liegen auch die Resultate der statistischen Analyse sehr nahe beieinander. Dies gilt vor allem für die grammatischen Variablen. Die Variablen, die bei beiden Ana- 
lysen berücksichtigt werden konnten, WORTART und GENUS, erreichen jeweils das Signifikanzniveau und die Bedeutung der jeweiligen Merkmalsausprägungen für die Ausprägung der abhängigen Variable (Dativ vs. kein_Dativ) stimmt in beiden Modellen überein. Auch die Bedeutung, die den soziolinguistischen Variablen beigemessen wird, ist bei beiden Datentypen weitestgehend deckungsgleich. So erreichen in beiden Modellen ALTER und GEBURTSLAND das veranschlagte Signifikanzniveau nicht, während M_L1_DEUTSCH jeweils als signifikanter Prädiktor integriert wird. Allerdings unterscheiden sich beide Modelle hinsichtlich der Variablen Geschlecht und V_L1_Deutsch. Diese sind bei den Fragebogendaten von Bedeutung, bei den Korpusdaten hingegen nicht. Zur Klärung der Frage, wie diese sich widersprechenden Ergebnisse zu interpretieren sind, muss zunächst ein Blick auf die Aussagekraft der Datentypen geworfen werden. Da die Korpusdaten ungesteuerten Sprachgebrauch in einer informellen Situation innerhalb der peer group oder der Familie dokumentieren, sind diese Daten sicher natürlicher und authentischer als die Fragebogendaten. Für die Fragebogendaten spricht allerdings, dass hier eine deutlich größere Gruppe von Personen berücksichtigt werden konnte und die Erkenntnisse somit aussagekräftiger sind mit Blick auf die Grundgesamtheit. Aus diesem Grund möchte ich keinen der beiden Datentypen bei der Interpretation der Ergebnisse vorrangig behandeln. Wenn man beide Ergebnisse gleichermaßen berücksichtigt, kann man zunächst festhalten, dass der Einfluss der Erstsprache des Vaters und des Geschlechts der SprecherInnen etwas unklar bleiben. Schlussfolgerungen sind aber dennoch möglich. Beim Vergleich der Variablen M_L1_DEuTsch und V_L1_DEuTsCH zeigt sich, dass die Erstsprache der Mutter ungeachtet des Datentyps relevant ist, während dies beim Vater nicht der Fall ist. M_L1_DEUTSCH ist demnach der zuverlässigere Prädiktor. Das kann als Indiz dafür gewertet werden, dass der Sprachgebrauch der Mutter prägender ist, was in Anbetracht der klassischen Rollenverteilung eine Erkenntnis ist, die sich soziolinguistisch durchaus gut erklären ließe. Darüber hinaus gilt hinsichtlich des Geschlechts der SprecherInnen, dass es nicht über beide Datentypen hinweg einen Effekt gibt. Dort aber, wo sich ein Effekt manifestiert, zeigt sich, dass weibliche Mitglieder der Community häufiger einen Dativ - und damit eine standardkonforme Variante - verwenden als männliche. Auch dieses Ergebnis lässt sich inhaltlich gut erklären bzw. fügt sich gut in bisherige soziolinguistische Ergebnisse ein. So sprechen im Allgemeinen Frauen bei zeitstabiler Variation tendenziell standardnäher als Männer (vgl. z. B. Tagliamonte 2012: 32). Evidenz für diesen Sachverhalt gibt es auch aus dem Namdeutschen: Hier verwenden Frauen tendenziell weniger namibiaspezifische Fremdwörter als Männer (vgl. Zimmer i. Ersch., Bracke i. Ersch.). 


\subsection{Soziolinguistische Variablen}

Ein wesentliches Ergebnis, das von der Analyse beider Datentypen gestützt wird, ist die Tatsache, dass das Namdeutsche hinsichtlich des Kasusgebrauchs als relativ variationsarm zu beschreiben ist. Zudem befindet sich das Namdeutsche in dieser Hinsicht sehr nah am deutschland-deutschen Standard. Dies gilt zum einen für die Verwendung des Dativs. Hier gibt es zwar eine nicht-marginale Schwankung, der Dativ wird in den entsprechenden syntaktischen Kontexten aber dennoch wesentlich häufiger verwendet als seine Alternativen. Zum anderen unterstützt der Gebrauch der anderen Kasus diese Beobachtung. Hier gibt es im Namdeutschen noch weniger Variation als beim Dativ. Hinzu kommt, dass das Dativ-Plural- $n$ konsequent verwendet wird und das Namdeutsche auch in dieser Hinsicht als ausgesprochen variationsarm $\mathrm{zu}$ beschreiben ist. Vor dem Hintergrund deutlicher Kasusschwankungen in anderen extraterritorialen Varietäten des Deutschen sind diese Beobachtungen durchaus bemerkenswert. Unabhängig von den Kontaktsprachen (vgl. z.B. Rosenberg 2003) und den Dialektmerkmalen der ausgewanderten Deutschsprachigen (vgl. z. B. Yager et al. 2015: 6) ist in diesen Varietäten zu beobachten, dass vor allem die Verwendung des Dativs erheblichen Schwankungen unterliegt und von teilweise massiven Abbautendenzen betroffen ist. Dies gilt bzw. galt (mitunter hat inzwischen ein Sprachwechsel stattgefunden) für deutschsprachige Communities in Argentinien (Yager et al. 2015), Australien (Clyne 2003: 124-130), Brasilien (u. a. Rosenberg 2016, 2018), Kansas (Keel 1994: 98), Oklahoma (Pulte 1970), Texas (u.a. Boas 2009a: 174-218, 2009b, 2011; Eikel 1949, 1967; Gilbert 1965; Guion 1996; Pulte 1970; Salmons 1983; 1994; Wilson 1960; Yager et al. 2015), Wisconsin (Yager et al. 2015), Russland (u. a. Rosenberg 2016; 2018), Südafrika (Franke 2008: 259-278; de Kadt 2001) und für Pennsylvania German (u. a. Huffines 1991, 1994; Louden 1988: 146-155, 1994; Van Ness 1994, 1996). Häufig sind sowohl die Kasusmarkierung am Pronomen/Artikel betroffen als auch das Dativ-Plural- $n$ als nominaler Kasusmarker, wobei letzteres mitunter schon sehr stark abgebaut ist, während Dative im Bereich der Artikel/Pronomen noch frequenter markiert werden (vgl. z. B. Rosenberg 2016: 193). Mit dem Sprachgebrauch in all diesen Communities hat das Namdeutsche gemein, dass - abgesehen vom weitestgehend obsoleten Genitiv - der Dativ am stärksten von Variation betroffen ist, während die Verwendung von Nominativ und Akkusativ stabil(er) ist. Allerdings sind in allen genannten Communities Wandel und/oder Variation beim Dativ deutlich stärker ausgeprägt.

Die Gründe für die Nähe zum Standarddeutschen sind vielfältig. Ein wesentlicher Faktor ist dabei sicher der Schulunterricht. Über diesen Aspekt geben auch die Korpusdaten Aufschluss. So hat der Faktor ScHULE in der oben beschrie- 
benen GLMM einen signifikanten Einfluss auf die Dativ-Verwendung. In Tabelle 8 ist der entsprechende Teil aus den oben vorgestellten Ergebnissen noch einmal wiederholt.

Tabelle 8: Der Einfluss des Faktors SCHULE

\begin{tabular}{llllll}
\hline & Estimate & Std. Error & z value & $\operatorname{Pr}(>|\mathbf{z}|)$ & \\
\hline SCHULE & \multicolumn{2}{l}{ Referenzlevel: DHPS } & & & \\
\hline Delta & 1.411645 & 0.559622 & 2.522 & 0.011653 & $*$ \\
englischsprachig & 1.08051 & 0.566845 & 1.906 & 0.056626 &. \\
Namib_High & 2.508723 & 0.581191 & 4.317 & $1.59 \mathrm{E}-05$ & $* \star$ \\
OSS & 0.564514 & 0.56661 & 0.996 & 0.319104 & \\
PSS & 1.593544 & 0.519115 & 3.07 & 0.002142 & $\star \star$ \\
sonstige & 0.340574 & 0.623664 & 0.546 & 0.585007 & \\
\hline
\end{tabular}

Das Referenzlevel für diesen Faktor ist DHPS. Die Wahrscheinlichkeit, dass ein Dativ verwendet wird, ist bei SprecherInnen, die diese Schule besuch(t)en, am höchsten, was daran zu erkennen ist, dass die Estimates der anderen Merkmalsausprägungen positiv sind und hier dementsprechend eine höhere Wahrscheinlichkeit von kein_Dativ vorliegt. Signifikant sind diese Unterschiede für die Merkmalsausprägungen Delta, Namib_High und PSS.

Dass sich die SprecherInnen, die die DHPS besuch(t)en, von anderen Schulen abheben und häufiger standardkonforme Varianten verwenden, ist wenig überraschend. Bei der DHPS handelt es sich um die einzige deutsche Auslandsschule in Namibia. Hier kann das Deutsche Internationale Abitur abgelegt werden, zahlreiche Lehrkräfte sind in Deutschland geboren und ausgebildet worden und im Gegensatz zu anderen Schulen wird nicht nur DaM bis in die höchsten Klassenstufen angeboten, sondern teilweise auch deutschsprachiger Unterricht in anderen Fächern (Shah \& Zappen-Thomson 2018). Neben diesen institutionellen Gegebenheiten spielen hier auch Spracheinstellungen eine wichtige Rolle. So manifestierte sich z. B. in den von uns geführten Interviews bei DHPS-SchülerInnen am deutlichsten eine Standardsprachideologie (orientiert am deutschlanddeutschen Standard), was auch durch darüberhinausgehende Beobachtungen bei informellen Gesprächen und Kommentare zur Datenerhebung gestützt wird. Folglich kann man davon ausgehen, dass dem deutschland-deutschen Standard - aus institutionellen und einstellungsbezogenen Gründen - an der DHPS eine besonders wichtige Rolle zukommt. Vor diesem Hintergrund ist es gut nachvollziehbar, dass die Wahrscheinlichkeit, dass ein (standardkonformer) Dativ verwendet wird, bei SprecherInnen mit DHPS-Hintergrund am höchsten ist. Dass 
es einen signifikanten Unterschied zwischen den beiden Windhoeker Schulen mit DaM-Unterricht gibt (Delta vs. DHPS), zeigt auch deutlich, dass hier die Art der Beschulung relevant ist und nicht etwa Stadt-Land-Unterschiede und/oder diatopische Variation zu beobachten sind.

Da Mitglieder der deutschsprachigen Community in der Regel (mindestens in der Primarstufe) eine Schule besuchen, an der DaM angeboten wird, sind in unserem Korpus kaum SprecherInnen vertreten, die überhaupt nicht auf Deutsch beschult wurden. Aus diesem Grund können keine systematischen Vergleiche angestellt werden, die in dieser Hinsicht differenzieren. Ungeachtet dessen liefern unsere Daten aber einen Hinweis auf die generelle Bedeutung des muttersprachlichen Schulunterrichts. Dass der Faktor ScHUlE einen signifikanten Einfluss hat, ist nicht nur aufschlussreich hinsichtlich zu beobachtender Differenzen innerhalb der Community. Vielmehr lässt sich aus dieser Tatsache auch ableiten, dass der Sprachgebrauch der namibischen SprecherInnen überhaupt durch schulische Einflüsse geprägt wird. Während die Rolle des Schulunterrichts gerade auch hinsichtlich der Kasusverwendung in anderen Communities nach wie vor diskutiert wird (vgl. z.B. Salmons 1994 und Boas 2009b), kann man meines Erachtens im Falle des namibischen Deutschen also davon ausgehen, dass die Beschulung ein Einflussfaktor mit Blick auf Variation und Wandel bzw. sprachliche Stabilität ist - und eine Erklärung für die relative Nähe des namibischen Deutschen zum Standarddeutschen.

Ein weiterer potentieller Einflussfaktor besteht darin, dass zahlreiche NamibierInnen häufigen und intensiven Kontakt mit Deutschland-Deutschen haben. Dieser resultiert häufig aus Tätigkeiten im Tourismusbereich, der eine wichtige Rolle in der namibischen Wirtschaft (und gerade auch in der deutschsprachigen Community) einnimmt oder auch aus Kontakt zu in Europa lebenden Verwandten. Die Häufigkeit des Kontakts (im Modell kategorisiert in nie, ein Mal im Jahr, ein Mal im Monat, ein Mal in der Woche und öfter) verfehlt innerhalb der Analyse der Korpusdaten das Signifikanzniveau allerdings knapp. Das schließt aber natürlich nicht aus, dass dieser Faktor bei anderen Phänomenen einen signifikanten Einfluss haben könnte.

Darüber hinaus spielen sicher auch deutschsprachige namibische Medien eine Rolle sowie Levelling im Varietätenkontakt in der Geschichte des namibischen Deutschen (zum Levelling vgl. Zimmer i. Dr.). Ferner ist auch die allgemeine Vitalität des namibischen Deutschen bedeutsam. Anders als in vielen anderen extraterritorialen Varietäten reduzieren sich die Domänen, in denen das Deutsche verwendet wird, hier nämlich nicht und Deutsch wird konsequent an folgende Generationen weitergegeben (vgl. z. B. Wiese et al. 2017; zur Registerdifferenzierung s. Wiese \& Bracke i. Ersch.). Namdeutsch kann folglich keineswegs als dying language bezeichnet werden, was hier insofern relevant ist, als z. B. 
Boas (2009b: 202) konstatiert: „One important property of dying languages is an increase in variability“.

Auch hinsichtlich der unterschiedlichen Generationen lassen sich beim Kasusgebrauch schließlich keine großen Unterschiede feststellen - bei beiden Datentypen erreicht die Variable ALTER das Signifikanzniveau nicht. Folglich ist der Kasusgebrauch auch über verschiedene Generationen hinweg im Namdeutschen weitgehend stabil. Demnach stützt der Kasusgebrauch Indizien aus dem Bereich der Lexik für vergleichsweise geringe sprachliche Unterschiede zwischen den Generationen (vgl. Zimmer i. Ersch.).

Somit lässt sich zunächst einmal festhalten, dass sich die Schwankungen beim Kasusgebrauch im Namdeutschen und anderen extraterritorialen Varietäten des Deutschen insofern ähneln, als vor allem der Dativ betroffen ist, während die anderen Kasus keine oder weniger Variation verursachen. ${ }^{10}$ Im Namdeutschen ist die Variation im Vergleich $\mathrm{zu}$ den anderen Varietäten aber weitaus weniger stark ausgeprägt. Hier scheint der Schulunterricht eine wichtige Rolle zu spielen. Demnach ist es wohl auch kein Zufall, dass es die deutschsprachigen Communities in Südafrika sind, die dem Namdeutschen hinsichtlich des Sprachgebrauchs am ähnlichsten sind: Hier gibt es zwar eine stärkere Variation als im Namdeutschen, im Vergleich zu anderen Varietäten (z. B. in Texas) ist aber auch hier die Schwankung vergleichsweise moderat und der Dativgebrauch ist (noch) nicht besonders stark von Abbautendenzen gekennzeichnet (Franke 2008: 268-269). Ein wichtiger Grund könnte auch hier der Schulunterricht sein, der in vielerlei Hinsicht vergleichbar ist mit dem Unterricht in Namibia (vgl. Zimmer 2019; zu Südafrika s. auch de Kadt 2001: 73).

\subsection{Grammatische Variablen}

Neben der Tatsache, dass vor allem im Dativ Schwankungen zu beobachten sind, gibt es weitere Parallelen zwischen dem Namdeutschen und anderen extraterritorialen Varietäten des Deutschen. Und zwar wird ein Dativ tendenziell eher an Pronomen ausgedrückt als in Nominalgruppen. Diese Generalisierung fußt auf einer breiten empirischen Grundlage, da in einer erstaunlich hohen Anzahl an Varietäten exakt diese Beobachtung gemacht wurde - hinsichtlich dieses Merkmals gibt es also eine bemerkenswerte Konsistenz über die verschiedenen

10 Erklärungsansätze für diesen Sachverhalt finden sich z. B. bei Boas (2009b: 367-369) und Rosenberg (2018: 271); s. außerdem Rosenberg (2016), der in diesem Zusammenhang auch die Relevanz natürlichkeitstheoretischer Konzepte diskutiert (zur Natürlichkeitstheorie s. einführend z. B. Dressler 2000). 
Communities hinweg. Tabelle 9 listet einige Publikationen, die dieses Phänomen beschreiben. ${ }^{11}$

Tabelle 9: Literaturüberblick: Kasusmarkierung (Pronomen vs. Artikel) in extraterritorialen Varietäten des Deutschen

\begin{tabular}{ll}
\hline extraterritoriale Varietät(en) (in): & dokumentiert (u. a.) in ... \\
\hline Argentinien & Yager et al. (2015) \\
Brasilien & Rosenberg (2018) \\
Indiana (USA) & Nützel (1993: 310-315) \\
Pennsylvania German & Reed (1948), Van Ness (1994) \\
Russland & Rosenberg (2018) \\
Südafrika & Franke (2008: 273), de Kadt (2001: 72) \\
Texas (USA) & Salmons (1994), Yager et al. (2015) \\
Unserdeutsch & Maitz, Lindenfelser \& Volker (i. Vorb.) \\
Wisconsin (USA) & Yager et al. (2015) \\
\hline
\end{tabular}

Als mögliche Gründe für dieses Phänomen wurden zahlreiche Beobachtungen ins Feld geführt (Boas 2009b; Rosenberg 2003, 2005, 2016, 2018; Salmons 1994; Yager et al. 2015). Im Folgenden werden einige dieser Aspekte etwas detaillierter besprochen. Im Zentrum stehen dabei diejenigen Faktoren, deren Allgemeingültigkeit mithilfe der Namdeutsch-Daten aufgrund spezifischer Merkmale (z.B. häufiger Gebrauch von Demonstrativpronomen, s. unten) evaluiert werden kann. Der folgende Überblick, der viele wesentliche Aspekte aus der Diskussion zusammenträgt, ist Rosenberg (2016: 198) entnommen (vgl. auch Rosenberg 2018: 270271).

Der Umstand der Resistenz der Pronominalflexion gegenüber dem Kasussynkretismus, der in der Nominalflexion zu beobachten ist, könnte durch folgende Umstände bedingt sein:

(a) Die hohe Frequenz der Pronomina könnte sie resistenter machen gegenüber sprachlichem Wandel: „the conserving effect of usage frequency“ (Haspelmath 2008: 204).

(b) Personalpronomina haben häufig belebte Referenten, die eine deutlichere morphologische Unterscheidung ihrer syntaktischen Rollen verlangen.

[...]

(e) Personalpronomina sind häufig Suppletivformen; diese werden vermutlich individuell und als ganze Einheiten lexikalisch gespeichert.

11 Ähnliche Beobachtungen gibt es auch abseits des Deutschen. So gilt auch für viele weitere germanische Varietäten, dass Pronomen tendenziell mehr Kasusunterscheidungen erlauben als Nominalgruppen (vgl. z. B. Harbert 2007: 105). 
Zunächst zur Frequenz: Hier stellt sich die Frage, mit welcher Wortart die Pronomen verglichen werden. Salmons (1994: 64) zieht explizit den Vergleich von Pronomen und Substantiven heran. Hier gibt es sicher einen deutlichen Frequenzunterschied, allerdings bleibt etwas unklar, inwiefern dieser relevant ist mit Blick auf die Kasusmarkierung, da Kasus im Gegenwartsdeutschen vor allem am Artikel ausgedrückt wird, während nominale Kasusmarker ausgesprochen selten sind (vgl. z. B. Wurzel 1991). Interessanterweise zeigt sich zumindest im Namdeutschen (im Gegensatz zu anderen Varietäten, vgl. z. B. Rosenberg 2016: 193), dass hinsichtlich des nominalen Kasusmarkers - $n$ im Dativ Plural keine Variation zu beobachten ist, während der Kasusausdruck am Artikel schwankt (s. oben). Aus meiner Sicht aussagekräftiger als der Frequenz-Vergleich zwischen Pronomen und Substantiven ist der Vergleich von Pronomen und Artikeln, da dies die beiden Wortarten sind, die hinsichtlich des Kasusausdrucks die Hauptlast tragen. Dabei ist zu beachten, dass die Frequenz von Pronomen (besonders von Personalpronomen) stark von der untersuchten Textsorte abhängt und z. B. in Zeitungstexten deutlich seltener ist als in Gesprächsdaten. Im DNam-Subkorpus, das die freien Gespräche enthält und für die vorliegende Untersuchung ausgewertet wurde, überwiegen dann auch die Pronomen gegenüber den Artikeln hinsichtlich der Tokenzahl (15.850 vs. 5282). Fokussiert man allerdings auf solche Strukturen, in denen im Namdeutschen (auch) ein Dativ verwendet wird (was auch in Anbetracht der vielen Suppletivformen angebracht zu sein scheint, s.u.), also indirekte Objekte und bestimmte Präpositionen, überwiegen Artikel: 1050 Artikel-Tokens vs. 755 Pronomen-Tokens, die sich auf vergleichbar viele Typen verteilen, nämlich 41 Artikel-Types (dem, einem, jedem usw.) und 32 PronomenTypes (jedem, mir, uns usw.). Demzufolge kann man hier keine höhere Frequenz der Pronomen nachweisen, was unter der Annahme, dass Artikel-Frequenz entscheidender ist als Substantivfrequenz, das Frequenz-Argument schwächt.

Der Faktor Belebtheit wird vor allem von Yager et al. (2015) prominent thematisiert. Die AutorInnen interpretieren Wandel und Variation so, dass die Kasusmarkierung umstrukturiert wird und sich ein System der differentiellen Objektmarkierung (DOM, vgl. z. B. Bossong 1985) herausbildet: „speakers are developing patterns of Differential Object Marking (DOM), following a hierarchy in which preferences are shown cross-linguistically for marking case on animate and definite arguments over inanimate and indefinite“ (Yager et al. 2015: 2). Dabei beziehen sie sich auf die erweiterte Belebtheitshierarchie. Eine Version dieser erweiterten Belebtheitshierarchie ist in (24) abgebildet (Croft 2003: 112). Neben dem semantischen Merkmal Belebtheit im engeren Sinne integriert diese Skala auch die Faktoren Referentialität und Definitheit (vgl. z. B. Ackermann 2018: 59-60). 
(24) Animacy: first, second-person pronouns < third-person pronoun < proper names $<$ human common noun $<$ nonhuman animate common noun $<$ inanimate common noun

Je höher ein Element auf dieser Skala angeordnet ist, desto größer ist die Wahrscheinlichkeit, dass dieses Element im Satz die semantische Rolle des Agens einnimmt. Der funktionale Vorteil von DOM besteht nun darin, dass hochbelebte Elemente (im Sinne der erweiterten Belebtheitshierarchie) morphologisch gesondert markiert werden, wenn der seltene Fall eintritt, dass ein solches Element eine Patiens-Rolle einnimmt und in einem Objektkasus verwendet wird (vgl. z. B. Dammel \& Gillmann 2014: 209-211). ${ }^{12}$

Aus der Annahme, dass in extraterritorialen Varietäten des Deutschen Umstrukturierungen in Richtung DOM zu erkennen sind, lassen sich folgende einfach zu testende Hypothesen ableiten (Yager et al. 2015: 4), die im Folgenden anhand der Namdeutsch-Daten erörtert werden:

- $\quad$ Pronouns should show case marking overfull NPs, e. g., mit mir but mit den Mann;

- Definite should show case marking over indefinite, e. g., less dative on ein-determiners (indefinite) than der-determiners (definite), so standard einem should be realized as ein/einen more often than dem as der/den or das.

- Animate should show case marking over inanimate, so humans and animals should show more dative determiners than physical objects.

Wenn man bei den Namdeutsch-Daten zwischen Pronomen und Artikeln unterscheidet, zeigt sich tatsächlich der vorhergesagte Effekt. Sowohl in den Korpusdaten als auch bei den Fragebogendaten wird bei Pronomen häufiger eine Dativmarkierung gewählt als bei Artikeln (vgl. Tabelle 10).

Tabelle 10: Dativmarkierung bei Pronomen vs. Artikeln

\begin{tabular}{llll}
\hline & & $\mathbf{n}$ & $\begin{array}{l}\text { Anteil } \\
\text { Dativmarkierung }\end{array}$ \\
\hline Korpus & Artikel & 1045 & $89,6 \%$ \\
& Pronomen & 742 & $97.6 \%$ \\
Fragebogen & Artikel & 1526 & $93,1 \%$ \\
& Pronomen & 385 & $96,4 \%$ \\
\hline
\end{tabular}

12 Typischerweise werden DOM-Effekte bei direkten Objekten beobachtet. Inwiefern in diesem Zusammenhang auch indirekte Objekte und somit die Dativmarkierung relevant sind, wird von Yager et al. (2015: 2) diskutiert. 
Allerdings lohnt sich hier ein genauerer Blick auf die verschiedenen Pronomentypen, die im Korpus dokumentiert sind (im Lückentext kommen fast ausschließlich Personalpronomen vor). Und zwar werden im Namdeutschen durchaus auch frequent Demonstrativpronomen zur Referenz auf Personen verwendet (vgl. (25)).

(25) ich hab schon monate nich mehr mit dem geredet (NAM116M1)

(26) ich hab noch nich mit ihm gesprochn (NAM155M2)

Dieser Pronomentyp ähnelt funktional stark den Personalpronomen (vgl. (26)). Hinsichtlich der erweiterten Belebtheitshierarchie gibt es dann auch keine Unterschiede zwischen Demonstrativpronomen und Personalpronomen (der 3. Person). Dennoch unterscheidet sich das Flexionsverhalten der beiden Typen. Während Personalpronomen in weniger als 1\% der Fälle vom Dativ abweichen, kommen bei den Demonstrativpronomen mehr alternative Formen vor (vgl. z. B. (27)). ${ }^{13}$ Dies zeigt sich auch in den Ergebnissen der GLMM, wie an Tabelle 11 abzulesen ist, die die relevanten Aspekte aus Tabelle 3 herausgreift.

(27) ich liebe brandy melville. kennt ihr das? das is like ihr müsst die auf instagram folgn (NAM092W1)

Tabelle 11: Der Einfluss des Faktors WORTART

\begin{tabular}{lllllll}
\hline & Estimate & Std. Error & z value & $\operatorname{Pr}(>|z|)$ & \\
\hline WoRTART & \multicolumn{7}{l}{ Referenzlevel: Definitartikel } & & & \\
\hline Demonstrativpronomen & -0.260308 & 0.474079 & -0.549 & 0.582949 & \\
Indefinitartikel & 0.891787 & 0.318712 & 2.798 & 0.005141 & $\star \star$ \\
Personalpronomen & -2.378641 & 0.814233 & -2.921 & 0.003485 & $\star \star$ \\
Possessivartikel & 0.797945 & 0.302527 & 2.638 & 0.00835 & $\star \star$ \\
sonstige & -0.419075 & 0.477093 & -0.878 & 0.37973 & \\
\hline
\end{tabular}

Es gibt einen signifikanten Unterschied zwischen Personalpronomen und Definitartikeln, wobei Personalpronomen die Wahrscheinlichkeit, dass kein_Dativ auftritt, deutlich senken. Der Unterschied zwischen Definitartikeln und Demonstrativpronomen ist hingegen nicht signifikant. Interessant an dieser Beobachtung ist die Tatsache, dass Demonstrativpronomen und Definitartikel formal beinahe identisch sind, während sich beide stark von Personalpronomen abheben. Das

13 Um die Lesbarkeit des Beispiels zu verbessern, wurden in Beispiel (27) Satzzeichen ergänzt. 
legt die Vermutung nahe, dass es vor allem formale Aspekte sind, die die Schwankung beeinflussen, weniger die Belebtheitshierarchie: Das Flexionsverhalten der formseitig ähnlichen, aber funktional unterschiedlichen Demonstrativpronomen und Definitartikel liegt sehr nahe beieinander, während das Flexionsverhalten der funktional ähnlichen, aber formseitig unterschiedlichen Pronomen sich signifikant unterscheidet. Weitere Ausführungen $\mathrm{zu}$ formseitigen Aspekten folgen weiter unten.

Tabelle 11 gibt auch Aufschluss bezüglich der zweiten Hypothese von Yager et al. (2015: 4). Definitartikel und Indefinitartikel unterscheiden sich signifikant voneinander. Indefinitartikel erhöhen im Vergleich die Wahrscheinlichkeit, dass kein_Dativ auftritt, sodass die Korpusdaten die Hypothese in diesem Aspekt eindeutig bestätigen.

Schließlich ist auch Belebtheit im engeren Sinne bzw. die Unterscheidung zwischen menschlichen, tierischen und unbelebten Denotaten von Bedeutung. Da weder bei der Analyse der Korpusdaten (s. S. 309) noch bei der differenzierteren Untersuchung dieses Faktors im Fragebogen (s. S. 317) die entsprechenden Variablen das Signifikanzniveau erreichten, muss man davon ausgehen, dass dieser Aspekt keine Rolle spielt. Diese Beobachtung fügt sich gut in die Ergebnisse von Yager et al. (2015), da auch in den von ihnen untersuchten Varietäten kein entsprechender Effekt nachgewiesen werden kann.

Mit Blick auf die Relevanz der Belebtheitshierarchie und eine mögliche Umstrukturierung des Kasussystems in Richtung DOM lässt sich nun resümieren, dass die Namdeutsch-Daten eher gegen eine in diese Richtung gehende Analyse sprechen, da Belebtheit im engeren Sinne überhaupt keinen Einfluss hat und die verschiedenen Pronomentypen sich nicht homogen verhalten. Letzteres deutet darauf hin, dass das Sonderverhalten der Personalpronomen formseitig motiviert ist und nicht durch deren Funktion. Lediglich die Unterschiede bzgl. der Definitheit entsprechen eindeutig den Hypothesen.

Die bereits angesprochenen formseitigen Eigenschaften von Pronomen und Artikeln sind relevant mit Blick auf den letzten von Rosenberg (2016: 198) genannten Aspekt, der hier besprochen werden soll: Personalpronomen sind häufig Suppletivformen. Diese Tatsache ist gut in den folgenden Paradigmen zu erkennen (Tabelle 12). 
Tabelle 12: Flexionsparadigmen der Personalpronomen (Standarddeutsch)

\begin{tabular}{llllll}
\hline & 1.P. & 2.P. & 3.P. & & \\
\hline Nom & ich & du & sie & er & es \\
Akk & mich & dich & sie & ihn & es \\
Dat & mir & dir & ihr & ihm & ihm \\
\hline Nom & wir & ihr & & sie & \\
Akk & uns & euch & & sie & \\
Dat & uns & euch & & ihnen & \\
\hline
\end{tabular}

Im Gegensatz dazu findet man in der Substantivflexion, die Salmons (1994: 65) und daran anknüpfend auch Rosenberg (2016: 198) mit der Flexion der Personalpronomen kontrastieren, überhaupt keine Suppletivformen. Die Kasusmarkierung erfolgt hier - wenn überhaupt - streng agglutinierend (z. B. mit den Kinder-n). Und auch die Flexion der Artikel, die meines Erachtens auch hier aussagekräftiger ist als die Substantivflexion, erfolgt agglutinierend (vgl. die Beispiele in Tabelle 13).

Tabelle 13: Flexionsparadigmen der Definit- und Indefinitartikel (Standarddeutsch)

\begin{tabular}{|c|c|c|c|c|c|c|}
\hline & \multicolumn{3}{|c|}{ Definitartikel } & \multicolumn{3}{|c|}{ Indefinitartikel } \\
\hline & Fem & Mask & Neut & Fem & Mask & Neut \\
\hline Nom & $d-i e$ & $d$-er & $d$-as & ein-e & ein & ein \\
\hline Akk & $d$-ie & $d$-en & $d$-as & ein-e & ein-en & ein \\
\hline Dat & $d$-er & $d$-em & $d$-em & ein-er & ein-em & ein-em \\
\hline Nom & & $d$-ie & & & - & \\
\hline Akk & & $d$-ie & & & - & \\
\hline Dat & & d-en & & & - & \\
\hline
\end{tabular}

Dass bei den suppletiven Personalpronomen so gut wie immer die Dativform verwendet wird und gleichzeitig die Demonstrativpronomen hinsichtlich des Kasusgebrauchs stärker den Artikeln ähneln, ist ein Indiz für die Relevanz der formseitigen Merkmale. Die Paradigmen von Definitartikel und Demonstrativpronomen sind identisch bis auf den Dativ Plural (Demonstrativpronomen: denen).

Den Unterschied zwischen suppletiven und nicht-suppletiven Formen erklärt Rosenberg (2016: 198) folgendermaßen, wobei die Erklärung wohl auch auf den Unterschied zwischen Artikeln und Pronomen ausgeweitet werden kann (vgl. hierzu auch Howe 1996: 69-70): 
Suppletivformen [...] werden vermutlich individuell und als ganze Einheiten lexikalisch gespeichert. Der Verlust von Kasusmarkierungen an Nomina, die in ,Dekomposition“ gespeichert werden, ist daher weniger „disruptiv“ (Salmons 1994: 64) als die Ersetzung ganzer lexikalischer Einträge. Neurolinguistische Untersuchungen weisen darauf hin, dass monomorphematische Wörter und polymorphematische Wörter mit irregulärer Wortbildung (ebenso wie weniger produktive morphologische Muster und semantisch weniger transparente Wörter) als ganze Einheiten lexikalisiert werden („Full Listing“; vgl. Cholewa 1993).

Auch Boas (2009b: 367) thematisiert formseitige Aspekte und hebt die phonologische Nähe von Dativ und Akkusativ in den Artikel-Paradigmen der Maskulina hervor (z.B. dem vs. den), die sich begünstigend auf den Zusammenfall der Formen (in Texas German) auswirke. Die phonologische Nähe der Formen sei aber keine hinreichende Erklärung für die zu beobachtende Variation im Kasusgebrauch und liefere z. B. keine Erklärung dafür, dass sich Akkusativ-Formen zuungunsten von Dativ-Formen ausbreiten - und nicht andersherum (ebd.).

Dennoch scheint es angemessen zu sein, der phonologischen Ähnlichkeit zwischen Akkusativ und Dativ eine gewisse Bedeutung in der Variation beizumessen. Die Namdeutsch-Daten sprechen insofern dafür, als hier Genus bei beiden Datentypen einen signifikanten Effekt hat: Bei Feminina (Dativ- und Akkusativform deutlich distinkt: z. B. der vs. die) werden z. B. mehr Dativformen verwendet als bei Maskulina (Ähnlichkeit der Formen: z. B. dem vs. den). Darüber hinaus sind Dativformen im Plural (Dativ- und Akkusativform deutlich distinkt: z. B. den vs. die) anteilsmäßig frequenter als im Singular (zumindest in Teilen liegt eine phonologische Ähnlichkeit vor).

Hinzu kommt, dass als Alternative zum Dativ offenbar solche Formen dispräferiert werden, die mit dem Nominativ synkretistisch sind. Das scheint die Übernahme der Akkusativform für den Dativ im Femininum (z. B. die und eine: Nominativ/Akkusativ) und im Plural (die: Nominativ/Akkusativ) zu hemmen. Aufschlussreich ist in diesem Zusammenhang auch der Definitartikel im Neutrum: Als Alternative zur Dativform tritt hier nämlich keineswegs die Akkusativ-Form das auf, die synkretistisch mit dem Nominativ ist, sondern den (vgl. (28)).

(28) da waren wir zwei wieder in den Zimmer und das ging immer so rum irgendwie

Solche Formen sind in zahlreichen extraterritorialen Varietäten des Deutschen belegt (vgl. z. B. Clyne 2003: 129, Rosenberg 2016: 186, Salmons 1994: 67). Sie wurden als Anzeichen einer Restrukturierung gedeutet, im Zuge derer Kasusoppositionen nicht nur abgebaut (vor allem Dativ vs. Akkusativ), sondern auch aufgebaut werden (Nominativ vs. Obliquus, vgl. Salmons 1994: 67). Indem eine Artikelform bei neutralen Substantiven auftritt, die mit ihrem maskulinen Pendant 
formgleich ist (zusätzlich zu dem im Dativ auch den als Alternative), wird dann auch die „+/-Feminin-Genus-Schranke“ gestärkt, die ein ganz wesentliches Merkmal der deutschen Morphologie darstellt (Nübling 2008: 299). Sie bezeichnet die Gemeinsamkeiten der Nicht-Feminina (Maskulina und Neutra) und deren deutliche Abgrenzung von den Feminina. Diese Opposition zeigt sich dann auch insgesamt bei den Kasusschwankungen im Namdeutschen, da Maskulina und Neutra wesentlich stärker betroffen sind als die Feminina.

\section{Fazit}

Wie die Ausführungen in den vorangegangenen Kapiteln gezeigt haben, gibt es bemerkenswerte Parallelen zwischen dem Namdeutschen und anderen extraterritorialen Varietäten des Deutschen. So schwankt im Namdeutschen - wie in einigen anderen Varietäten auch - vor allem die Dativmarkierung, während Nominativ und Akkusativ durch größere Stabilität gekennzeichnet sind. Bei der Variation im Dativ ist zu beobachten, dass bestimmte Wortarten (z. B. Personalpronomen) weniger Variation unterliegen als andere (z. B. Indefinitartikel), wofür es auch zahlreiche Beispiele aus anderen Varietäten gibt. Solche Gemeinsamkeiten deuten darauf hin, dass sich hier Tendenzen und Prinzipien manifestieren, die varietäten- und sprachübergreifend wirksam sind.

Gleichzeitig gibt es auch Spezifika des Namdeutschen. Auffällig ist hier in erster Linie (zumindest die Grammatik betreffend) die Nähe zum Standarddeutschen. Einige potentielle Gründe für diesen Sachverhalt konnten hier in die Analyse einbezogen werden. Dabei hat sich gezeigt, dass besonders die Beschulung bedeutsam ist. Unter den sonstigen Faktoren haben sich in erster Linie diejenigen als einflussreich herausgestellt, die formseitig relevant sind (Genus, Numerus, Wortart).

Insgesamt hat sich bewahrheitet, dass ein komplexes Geflecht an verschiedenen (soziolinguistischen und grammatischen) Faktoren die Variation bei der Dativmarkierung beeinflusst und dass die Beschreibung und die Analyse dieses Faktoren-Geflechts mithilfe eines multifaktoriellen Modells einerseits das Verständnis und die Dokumentation des Namdeutschen voranbringen und andererseits auch einen Beitrag zur allgemeinen Diskussion um Schwankungen im Bereich der Kasusmarkierung leisten können.

Danksagung: Meine Arbeit wurde gefördert durch die Deutsche Forschungsgemeinschaft (DFG) - WI 2155/9-1; SI 750/4-1. Ich bedanke mich außerdem bei den namibischen Gewährspersonen für deren große Kooperationsbereitschaft, bei Anika Kroll-Tjingaete, Britta Stuhl und Victoria Weden für die Unterstützung bei 
der Datenerhebung und -aufbereitung sowie bei den anonymen GutachterInnen für ihre Kommentare.

\section{Literatur}

Ackermann, Tanja. 2018. Grammatik der Namen im Wandel. Diachrone Morphosyntax der Personennamen im Deutschen. (Studia Linguistica Germanica 134). Berlin \& Boston: De Gruyter.

Baayen, R. H. 2008. Analyzing Linguistic Data. A Practical Introduction to Statistics Using R. Cambridge: Cambridge University Press.

Backhaus, Klaus, Bernd Erichson, Wulff Plinke \& Rolf Weiber. 2003. Multivariate Analysemethoden. 10. Aufl. Berlin \& Heidelberg: Springer.

Bates, Douglas, Martin Mächler, Ben Bolker \& Steve Walker. 2015. Fitting Linear Mixed-Effects Models Using Ime4. Journal of Statistical Software 67. 1-48.

Boas, Hans C. 2009a. The Life and Death of Texas German. (Publications of the American Dialect Society 93). Durham, NC: Duke University Press.

Boas, Hans C. 2009b. Case loss in Texas German. In Jóhanna Barðdal \& Shobhana L. Chelliah (Hrsg.), The Role of Semantic, Pragmatic and Discourse Factors in the Development of Case, 347-376. Amsterdam \& Philadelphia: Benjamins.

Boas, Hans C. 2011. Some Consequences of Case Loss in Texas German. In Corey Yoquelet (Hrsg.), Proceedings of the tewnty-ninth Annual Meeting of the Berkely Linguistics Society, 43-54. Berkely: Berkeley Linguistics Society.

Böhm, Michael Anton. 2003. Deutsch in Afrika: die Stellung der deutschen Sprache in Afrika vor dem Hintergrund der bildungs- und sprachpolitischen Gegebenheiten sowie der deutschen auswärtigen Kulturpolitik. (Duisburger Arbeiten zur Sprach- und Kulturwissenschaft 52). Frankfurt am Main: Lang.

Bossong, Georg. 1985. Differentielle Objektmarkierung in den Neuiranischen Sprachen. Tübingen: Narr.

Bracke, Yannic. i. Ersch. Namibian German and gender: A corpus study on the use of transferred lexical items. In Christian Zimmer \& Horst J. Simon (Hrsg.), German(ic) in Language Contact: Grammatical and Sociolinguistic Dynamics.

Cholewa, Jürgen. 1993. Störungen der lexikalisch-morphologischen Wortverarbeitung bei Aphasie: Ein Literaturüberblick. Neurolinguistik 7. 105-126.

Clyne, Michael. 2003. Dynamics of Language Contact. Cambridge: Cambridge Univ. Press.

Croft, William. 2003. Typology and Universals. 2. Aufl. Cambridge: Cambridge University Press.

Dammel, Antje \& Melitta Gillmann. 2014. Relevanzgesteuerter Umbau der Substantivflexion im Deutschen. Beiträge zur Geschichte der deutschen Sprache und Literatur (PBB) 136. 173-229.

Deppermann, Arnulf, Stefan Kleiner \& Ralf Knöbl. 2013. „Standard usage“ - towards a realistic conception of spoken standard German. In Peter Auer, Javier Caro Reina \& Göz Kaufmann (Hrsg.), Language Variation - European Perspectives IV: Selected papers from the Sixth International Conference on Language Variation in Europe (ICLaVE 6), Freiburg, June 2011, 83-116. (Studies in Language Variation 14). Amsterdam \& Philadelphia: Benjamins. 
Dressler, Wolfgang U. 2000. Naturalness. In Geert Booij, Christian Lehmann \& Joachim Mugdan (Hrsg.), Morphologie, 288-296. (Handbücher zur Sprach- und Kommunikationswissenschaft 17.1). Berlin \& New York: De Gruyter.

Duden-Grammatik. 2016. Die Grammatik. 9. Aufl. (Duden 4). Berlin: Dudenverlag.

Eikel, Fred Jr. 1949. The use of cases in New Braunfels German. American Speech 24. 278-281.

Eikel, Fred Jr. 1967. New Braunfels German: Part III. American Speech 42. 83-104.

Eisenberg, Peter. 2013. Grundriss der deutschen Grammatik. Band 2: Der Satz. 4. Aufl. Stuttgart \& Weimar: Metzler.

Franke, Katharina. 2008. “We call it Springbok-German!”: Language Contact in the German Communities in South Africa. Monash University.

Gilbert, Glenn G. 1965. Dative vs. accusative in the German dialects of Central Texas. Zeitschrift für Mundartforschung 32. 288-296.

Guion, Susan. 1996. The death of Texas German in Gillespie County. In lain Clarkson \& P. Sture Ureland (Hrsg.), Language Contact across the North Atlantic, 443-463. (Linguistische Arbeiten 359). Tübingen: Niemeyer.

Harbert, Wayne. 2007. The Germanic languages. Cambridge: Cambridge University Press.

Haspelmath, Martin. 2008. Creating Economical Morphosyntactic Patterns in Language Change. In Jeff Good (Hrsg.), Linguistic Universals and Language Change, 185-214. Oxford: Oxford University Press.

Hosmer, David W. \& Stanley Lemeshow. 2000. Applied Logistic Regression: Hosmer/Applied Logistic Regression. Hoboken: Wiley. doi:10.1002/0471722146.

Howe, Stephen. 1996. The Personal Pronouns in the Germanic Languages. A Study of Personal Pronoun Morphology and Change in the Germanic Languages from the First Records to the Present Day. (Studia Linguistica Germanica 43). Berlin \& New York: De Gruyter.

Huffines, Marion L. 1991. Translation: A vehicle for change? Evidence from Pennsylvania German. American Journal of Germanic Linguistics and Literatures 3. 175-193.

Huffines, Marion L. 1994. Directionality of language influence: The case of Pennsylvania German and English. In Nina Berend \& Klaus J. Mattheier (Hrsg.), Sprachinselforschung: Eine Gedenkschrift für Hugo Jedig, 47-58. Frankfurt am Main: Lang.

de Kadt, Elizabeth. 2001. "You still speak German?”: Teenage language skills in a Germanspeaking community. In Tom Ammerlaan (Hrsg.), Sociolinguistic and Psycholinguistic Perspectives on Maintenance and Loss of Minority Languages, 61-72. Münster: Waxmann.

Keel, William D. 1994. Reduction and loss of case marking in the noun phrase in GermanAmerican speech islands: Internal development or external interference? In Nina Berend \& Klaus J. Mattheier (Hrsg.), Sprachinselforschung: Eine Gedenkschrift für Hugo Jedig, 93-103. Frankfurt am Main: Lang.

Levshina, Natalia. 2015. How to Do Linguistic with R. Data Exploration and Statistical Analysis. Amsterdam \& Philadelphia: Benjamins.

Louden, Mark L. 1988. Bilingualism and Syntactic Change in Pennsylvania German. Cornell University.

Louden, Mark L. 1994. Syntactic change in multilingual speech islands. In Nina Berend \& Klaus J. Mattheier (Hrsg.), Sprachinselforschung: Eine Gedenkschrift für Hugo Jedig, 73-91. Frankfurt am Main: Lang.

Maitz, Péter \& Stephan Elspaß. 2013. Zur Ideologie des „Gesprochenen Standarddeutsch“. In Jörg Hagemann, Wolf Peter Klein \& Sven Staffeldt (Hrsg.), Pragmatischer Standard, 35-48. Tübingen: Stauffenburg. 
Maitz, Péter, Siegwalt Lindenfelser \& Craig Alan Volker. i. Vorb. Unserdeutsch (Rabaul Creole German), Papua New Guinea. In Hans C. Boas, Ana Deumert, Mark L. Louden \& Péter Maitz (Hrsg.), Varieties of German Worldwide. Oxford: Oxford University Press.

Nübling, Damaris. 2008. Was tun mit Flexionsklassen? Deklinationsklassen und ihr Wandel im Deutschen und seinen Dialekten. Zeitschrift für Dialektologie und Linguistik 75. 282-330.

Nützel, Daniel. 1993. Case loss and morphosyntactic change in Haysville East Franconian. In Joseph C. Salmons (Hrsg.), The German Language in America, 1683-1991, 307-321. Madison: Max Kade Institute for German-American Studies.

Pulte, William Jr. 1970. An analysis of selected german dialects of northern Texas and Oklahoma. In Glenn G. Gilbert (Hrsg.), Texas Studies in Bilingualism, 105-141. Berlin: De Gruyter.

Reed, Caroll E. 1948. A survey of Pennsylvania German morphology. Modern Language Quarterly 9. 322-342.

Rosenberg, Peter. 2003. Comparative speech island research: Some results from studies in Russia and Brazil. In William Keel \& Klaus J. Mattheier (Hrsg.), Deutsche Sprachinseln weltweit: Interne und externe Perspektiven - German Language Varieties Worldwide: Internal and external Perspectives, 199-238. Frankfurt am Main: Lang.

Rosenberg, Peter. 2005. Language island research: the traditional framework and some sociolinguistic questions. In Peter Auer, Frans Hinskens \& Paul Kerswill (Hrsg.), Dialect Change. Convergence and Divergence in European Languages, 221-235. Cambridge: Cambridge University Press.

Rosenberg, Peter. 2016. Regularität und Irregularität in der Kasusmorphologie deutscher Sprachinselvarietäten (Russland, Brasilien). In Andreas Bittner \& Klaus-Michael Köpcke (Hrsg.), Regularität und Irregularität in Phonologie und Morphologie: Diachron, kontrastiv, typologisch. (Lingua Historica Germanica Band 13). Berlin \& Boston: De Gruyter.

Rosenberg, Peter. 2018. Überflutete Sprachinseln: Sprachvariation, Sprachwechsel und Sprachwandel in deutschen Sprachinseln in Russland und Brasilien. In Alexandra N. Lenz \& Albrecht Plewnia (Hrsg.), Variation - Normen - Identitäten, 263-282. Berlin \& Boston: De Gruyter.

Salmons, Joseph C. 1983. Issues in Texas German language maintenance and shift. Monatshefte 75. 187-196.

Salmons, Joseph C. 1994. Naturalness and morphological change in Texas German. In Nina Berend \& Klaus J. Mattheier (Hrsg.), Sprachinselforschung: Eine Gedenkschrift für Hugo Jedig, 59-73. Frankfurt am Main: Lang.

Schmidt, Jürgen Erich, Joachim Herrgen \& Roland Kehrein (Hrsg.). 2008 ff. a. Regionalsprache. de (REDE). Forschungsplattform zu den modernen Regionalsprachen des Deutschen. Bearbeitet von Dennis Bock, Brigitte Ganswindt, Heiko Girnth, Simon Kasper, Roland Kehrein, Alfred Lameli, Slawomir Messner, Christoph Purschke, Anna Wolańska. Marburg: Forschungszentrum Deutscher Sprachatlas.

Schmidt, Jürgen Erich, Joachim Herrgen \& Roland Kehrein (Hrsg.). 2008 ff. b. Dialekteinteilung nach Wiesinger (1983). Abzeichnung der Karte 47,4 in Wiesinger, Peter (1983): Die Einteilung der deutschen Dialekte. In: Besch, Werner [u. a.] (Hrsg.): Dialektologie. Ein Handbuch zur deutschen und allgemeinen Dialektforschung. 2. Halbband. (= Handbücher zur Sprach- und Kommunikationswissenschaft. 1.2) Berlin/New York. <www.regionalsprache.de>.

Schweizer, Harro. 1982. Deutsche Sprache unter der Apartheid. Zeitschrift für Sprachwissenschaft 2. 201-241. 
Shah, Sheena \& Marianne Zappen-Thomson. 2018. German in Namibia. In Corinne A. Seals \& Sheena Shah (Hrsg.), Heritage Language Policies around the World, 128-147. Abingdon \& New York: Routledge.

Tagliamonte, Sali A. 2012. Variationist Sociolinguistics. Change, Observation, Interpretation. (Language in Society 39). Malden: Wiley-Blackwell.

Thieroff, Rolf \& Petra M. Vogel. 2012. Flexion. 2. Aufl. (Kurze Einführungen in die germanistische Linguistik 7). Heidelberg: Winter.

Van Ness, Silke. 1994. Pennsylvania German. In Ekkehard König \& Johan Van der Auwera (Hrsg.), The Germanic Languages, 420-438. London: Routledge.

Van Ness, Silke. 1996. Case syncretism in Pennsylvania German: Internal or external forces at play? American Journal of Germanic Linguistics and Literatures 8.1-17.

Westpfahl, Swantje. 2014. STTS 2.0? Improving the Tagset for the Part-of-Speech-Tagging of German Spoken Data. LAW VIII - The 8th Linguistic Annotation Workshop, 1-10.

Wiese, Heike \& Yannic Bracke. i. Ersch. Registerdifferenzierung im Namdeutschen: Informeller und formeller Sprachgebrauch in einer vitalen Sprechergemeinschaft. In Csaba Földes (Hrsg.), Kontaktvarietäten des Deutschen im Ausland. Tübingen: Narr.

Wiese, Heike, Horst J. Simon, Christian Zimmer \& Kathleen Schumann. 2017. German in Namibia: A vital speech community and its multilingual dynamics. Language \& Linguistics in Melanesia (Sonderheft: Language Contact in the German Colonies: Papua New Guinea and beyond; hg. v. Péter Maitz \& Craig A. Volker). 221-245.

Wilson, Joseph. 1960. The Texas German of Lee and Fayette Counties. Rice Institute Pamphlet 47. 83-98.

Wurzel, Wolfgang Ullrich. 1991. Genitivverwirrung im Deutschen - wie regulär ist morphologischer Wandel? In Norbert Boretzky, Thomas Stolz, Benedikt Jeßing \& Werner Enninger (Hrsg.), Sprachwandel und seine Prinzipien, 168-181. (Bochum-Essener Beiträge zur Sprachwandelforschung 14). Bochum: Brockmeyer.

Yager, Lisa, Nora Hellmold, Hyoun-A Joo, Michael Putnam, Eleonora Rossi, Catherin Stafford \& Joseph C. Salmons. 2015. New structural patterns in moribund grammar: case marking in heritage German. Frontiers in Psychology 6:1716. 1-9.

Zappen-Thomson, Marianne. 2019. Stützung des Spracherhalts bei deutschsprachigen Minderheiten: Namibia. In Ulrich Ammon \& Gabriele Schmidt (Hrsg.), Förderung der deutschen Sprache weltweit, 517-530. Berlin \& Boston: De Gruyter.

Zimmer, Christian. 2019. Deutsch als Minderheitensprache in Afrika. In Joachim Herrgen \& Jürgen Erich Schmidt (Hrsg.), Sprache und Raum - Deutsch. Ein internationales Handbuch der Sprachvariation, 1176-1190. (Handbücher zur Sprach- und Kommunikationswissenschaft 30.4). Berlin \& Boston: De Gruyter Mouton.

Zimmer, Christian. i. Dr. Siedlungsgeschichte und Varietätenkontakt: Zur Entstehung des Namdeutschen. Zeitschrift für Dialektologie und Linguistik.

Zimmer, Christian. i. Ersch. Linguistic variation and age of speakers in Namibian German: loan word usage in „Wenker sentences“. In Hans C. Boas (Hrsg.), German Abroad: Comparative Perspectives on language Contact.

Zimmer, Christian, Heike Wiese, Horst J. Simon, Marianne Zappen-Thomson, Yannic Bracke, Britta Stuhl \& Thomas Schmidt. 2020. Das Korpus Deutsch in Namibia (DNam): Eine Ressource für die Kontakt-, Variations- und Soziolinguistik. Deutsche Sprache 2020. 210-232. 